\title{
Preparation and Unequivocal Identification of Chromophores-Substituted Carbosilane Dendrimers up to $7^{\text {th }}$ Generations
}

\author{
Chungkỵun Kim, ${ }^{*}$ Hyojung Kim, Myeong-Jin Oh, and Jang-Hwan Hong*
}

\author{
Department of Chemistry, Dong-1 Liniversity, Busan 604-714, Korea. "E-mail: ckkimadau.ac.kr \\ ${ }^{\dagger}$ Department of Nanopolymer Material Engineering. Pai Chai Unwersity. Daejeon $302-735$. Korea \\ Received Jantary 8, 2009, Accepted Harch 3, 2009
}

\begin{abstract}
Bis(phenylethynyl)dimethylsilane is branched by the hydrosilation of the phenylethynyl group with dichloromethylsilane, and then the resulting chlorosilane is reacted with lithium phenylacetylide to give the $\mathrm{I}^{\text {st }}$ generation. The same hydrosilation and alkynylation are repeated to obtain the $7^{\text {th }}$ generation. In addition peripheral $\mathrm{Si}-\mathrm{Cl}$ moiety of the seven kind generation dendrimers are reacted with alcoholic moiety of 9-hyddroxymethylanthracene and 2-(2-hydroxyphenyl)benzoxazole group in the presence of TMEDA. Then three kinds of carbosilane dendrimers are prepared from the $1^{\text {st }}$ to the $7^{\text {th }}$ generations, the $7^{\text {th }}$ generation of each dendrimer has 256 phenylethynyl, 2569-anthracenylmethoxy, or 1282-(2-phenoxy )benzosazole groups. Each synthesized dendrimer is unequivocally characterized by ${ }^{1} \mathrm{H}$ and ${ }^{13} \mathrm{C} N \mathrm{NR}$, elemental analy sis, MALDI-MS, GPC, and PL (photoluminescence). Characteristically PDI (Polydisperse Index) values of the dendrimers' peak in GPC are in the range of 1.00 1.07, which indicates that each generation of carbosilane is in unified distribution. PL spectra of phenylethynyl and 9anthracenemethoxy group substituted dendrimers show no significant change with increasing the generation from the $1^{\text {st }}$ to the $7^{\text {th }}$. However, the PL spectra of $2-(2$-phenoxy)benzoxazole group substituted dendrimers show a blue-shift trend with increasing the generation from the $1^{\text {st }}$ to the $7^{\text {th }}$.
\end{abstract}

Key Words: Dendrimer. Silane. Siloxane. Photoluminescence. GPC

\section{Introduction}

Since Vögtle reported the first cascade synthesis ${ }^{1-\hat{A}}$ new homologous dendritic molecules have been prepared. ${ }^{3-5}$ Nowadays the application of dendrimer becomes more important issue for many researchers ${ }^{6-13}$ Defect free dendritic structures (dendrons or dendritic wedges are structurally monomolecular or unimolecular in composition) are available by the using the limited number of reactions under controlled reaction conditions of temperature. pressure. and large excess of reagents. ${ }^{1+1 \%}$ In the case of general polymer it is well known that every chain reaction provides a mixture of molecular linear polymers.

It has been a controversial subject to confirm the moleculelarity of dendrimer (molecular purity or stnictural defect). especially that of higher generation dendrimer due to the self-similarity inherent in the dendritic architecture. ${ }^{15.18}$ For lower generation dendrimer, the molecular weight of which is around thousands Daltons, the unimolecularity has been confirmed by mass spectroscopy. ${ }^{18-19}$ MS (Mass Spectroscopy) is useful to determine the molecular weight of lower generation dendrimer with its molecular ion peak and fragmentation pattern. but it still gives no decisive information for its structural defect of higher generations. ${ }^{\text {?ci }}$ In this reason GPC (The gel permeation chromatograply) is used to determine the unimolecularity of dendrimer. ${ }^{10.71}$

Herein. novel carbosilane dendrimers $\left(\mathrm{I}^{\text {st }}\right.$ to $7^{\text {th }}$ generation). having chromophores of phenylethynyl. antracenyl. and benzoxazoyl groups on the periphery, are synthesized and characterized by ${ }^{1} \mathrm{H}$ and ${ }^{13} \mathrm{C}$ NMR. MALDI-MS and elemental analysis. their unimoleculelarities are confirmed by GPC. Additionally dendritic effects of the chromophores of them are studied by PL (photoluminescence). The purpose of the article is observation of unimolecularities of these carbosilane dendrimers and dendritic effect of them is studied by PL (photoluminescence) spectroscopy:

\section{Experimental}

All reactions were carried out under a dried nitrogen atmosphere attached to vacuun line. NMR spectra were recorded on a Bnuker AC 200. UV spectra were measured by HP 8452A diode array ultraviolet visible spectrophotometer. The GPC was performed in THF at $25^{\circ} \mathrm{C}$ with Waters 515 HPLC pump and Waters 2410 Refractive Index Detector connected with 3 colunns (Ultrastyragel $0.78 \times 30 \mathrm{~cm} ; 10^{2}$, $10^{4}$ and $10^{5}$ ). which was calibrated by narrow molecular weight of polystyrene standard. Elemental analyses were carried out at KBSI in Daegu. Korea. The following abbreviations are used: PA refers to phenyl ethylenyl. AN refers to anthracene. BO refers to benzoxazoyl group. TMEDA refers to tetramethylethylenediamine. In the representation of $n G\left[2,2^{n-1} \cdot 1\right]-2^{n} X$ and $n G\left[2,2^{n-1} \cdot 2\right]-2^{n+1} X$; the $n^{\text {th }} G$ refers to generation number. the first 2 in bracket refers to the number of branch in core. the $2^{\text {nt- }-1}$ refers to the number of branches in inside and last 1 or 2 in the bracket refers to the number of functional groups on the periphery and last $2^{n)} \mathrm{X}$ or $2^{\mathrm{n}+1} \mathrm{X}$ refers to the number of $X$ functional groups on the whole periphery. Hydrosilation of triple bonds on dendritic periphery and alkynylation by lithum phenylacetylide were described in the previous reports. ${ }^{15}$

Dichloromethylsilane is used for the hydrosilation of the preparation of $n \mathrm{G}\left[2.2^{n \cdot 1}, 2\right]-2^{n-1} \mathrm{PA}$ and $\mathrm{nG}\left[2.2^{n \cdot 1}, 2\right]-2^{n-1} \mathrm{AN}$, 
chlorodimethylsilane is used for the hydrosilation of the preparation of $\mathrm{nG}\left[2.2^{\mathrm{n} \cdot \mathrm{l}}, 1\right]-2^{\mathrm{n}} \mathrm{BO}$.

\section{(1) Synthesis of $n G\left[2,2^{n-1}, 2\right]-2^{n+1} P A$ type dendrimers.}

1G[2,0,2]-4PA: $55 \mathrm{~mL}(55 \mathrm{mmol})$ of lithium phenylacetylide ( $\mathrm{I} \mathrm{M}$ in THF) was added to the solution of $5.65 \mathrm{~g}$ (11.52 imol) of $1 \mathrm{G}[2,0,2]-4 \mathrm{Cl}$ in $50 \mathrm{~mL}$ of THF at room temperature. Then, the reaction mixture was refluxed for 2 hrs. After removing solvent from the mixture. toluene was added to the residue. Lithium chloride salt was removed by filtration with continual adding of activated charcoal until to remove the brown color. $1 G[2,0,2]-4$ PA was purified by silica gel chromatography with mixed solvent of toluene and hexane $(1: 1)$. Yield: $7.79 \mathrm{~g}(89 \%, 11.52 \mathrm{mmol})$ as light yellow to white powder. ${ }^{1} \mathrm{H}-\mathrm{NMR}$ (ppm. $\mathrm{CDCl}_{3}$ ): $\delta-0.38$ (s. $6 \mathrm{H}$. SiMe. G0). 0.40 (s. $6 \mathrm{H} . \mathrm{SiMe}$. Gl). $6.7 \mathrm{l}$ (s. $2 \mathrm{H} . \mathrm{CH}=\mathrm{C}$ ). $7.12 \sim 7.45$ (m. $30 \mathrm{H}, \mathrm{Ph} . \mathrm{GO} \sim \mathrm{Gl}$ ). ${ }^{13} \mathrm{C}-\mathrm{NMR}$ (ppm. $\mathrm{CDCl}_{3}$ ): $\delta-0.98$ (SiMe. G0). -0.67 ( (SiMe. Gl) $148.52,157.96(\mathrm{C}=\mathrm{C}) .89 .22,107.61$ $(\mathrm{C}=\mathrm{C}) .122 .70 .122 .77 .132 .12$ (Pl, Gl). 126.41. 128.21. 128.89. 143.21 (Ph. G0). Anal. Calc. for $\mathrm{C}_{52} \mathrm{H}_{44} \mathrm{Si}_{3}$ (753.2): C. $82.86 \%$. H. $5.84 \%$. Found: C. $82.82 \%$, H, $5.82 \%$. MALDITOF mass: $775.31(\mathrm{M}+\mathrm{Na})^{+}$. UV/Vis, $\lambda_{\text {max }} 253 \mathrm{~nm}, \varepsilon_{\text {max }} 0.64$ $\times 10^{5}$. GPC: PDI value $\left(\mathrm{M}_{1 *} / \mathrm{M}_{t 3}\right) .1 .01$ (753/739): Retention time (Rt). $19.66 \mathrm{~min}$.

2G[2,2,2]-8PA: $4.5+\mathrm{g}(3.74 \mathrm{mmol})$ of $2 \mathrm{G}[2.2 .2]-8 \mathrm{Cl}$ and $40 \mathrm{~mL}(40 \mathrm{mmol}$ ) of lithium phenylacety lide were used by the same procedure as the above for $1 \mathrm{G}[2,0,2]-4 \mathrm{PA}$. The mixture was refluxed for $5 \mathrm{hrs}$. Yield: $5.74 \mathrm{~g}(89 \%, 3.30 \mathrm{mmol})$ as yellow glassy material. 'H-NMR (ppm, $\mathrm{CDCl}_{3}$ ): $\delta-0.70$ (s. 6H. SiMe, G0). $-0.5 \mathrm{I}$ (s. 6H. SiMe. Gl). 0.39 (s. 12H. SiMe. G2). 6.10 (s. 2H. $\mathrm{CH}=\mathrm{C} . \mathrm{G} 0) .6 .71$ (s. $+\mathrm{H} . \mathrm{CH}=\mathrm{C} . \mathrm{Gl}) \cdot 6.90 \sim$ 7.47 (m. 70H. Ph. G0 G2) ${ }^{13} \mathrm{C}-\mathrm{NMR}\left(\mathrm{ppm}, \mathrm{CDCl}_{3}\right.$ ): $\delta-3.06$ (SiMe. G0). -0.53 (SiMe, G1), 0.95 (SiMe, G2). 144.91, 162.68 $(\mathrm{C}=\mathrm{C} . \mathrm{G} 0), 145.89 .158 .40(\mathrm{C}=\mathrm{C}, \mathrm{Gl}) .89 .2+, 107.52(\mathrm{C}=\mathrm{C})$. $125.90,127.59 .128 .12 .128 .19,128.8+(\mathrm{Pl}, \mathrm{GO} \sim \mathrm{Gl}) .126 .43$. 122.71. 127.72.132.10 (Ph, G2). Anal. Calc. for $\mathrm{C}_{12} \mathrm{H}_{100 \mathrm{~S}} \mathrm{Si}$ (1738.7): C. $82.82 \%$. H. 5.75\%. Found: C. $82.40 \%$. H. $5.84 \%$. MALDI-TOF mass: $1761.83(\mathrm{M}+\mathrm{Na})^{+}$. UV/Vis, $\lambda_{\text {maxi }} 253 \mathrm{~mm}$. $\varepsilon_{\text {max }} 1.12 \times 10^{5}$. GPC: PDI value $\left(\mathrm{M}_{w} / \mathrm{M}_{n}\right)$. 1.01 (1209/ 1188): Rt. $18.76 \mathrm{~min}$.

3G[2,2,2,2]-16PA: $4.79 \mathrm{~g}(1.87 \mathrm{mmol})$ of $3 \mathrm{G}[2.2,2,2] 16 \mathrm{Cl}$ and $40 \mathrm{~mL}(40 \mathrm{mmol})$ of lithium phenylacetylide were used by the same procedure as the above for $1 \mathrm{G}[2.0 .2]-4 \mathrm{PA}$. The mixture refluxed for $10 \mathrm{hrs}$. Yield: $5.27 \mathrm{~g} \mathrm{(76 \% .1.42 \textrm {mmol } )}$ as yellow glassy material. ${ }^{1} \mathrm{H}-\mathrm{NMR}$ (ppm, $\mathrm{CDCl}_{3}$ ): $\hat{o}-0.76$ (s. $6 \mathrm{H}$. SiMe. G0), -0.61 (s, 6H, SiMe, G1), -0.80 (s. 12H, SiMe. G2). $0.3+$ (s. 2+H. SiMe. G3). 5.93 (s. 2H. CH=C. G0). 6.03 $(\mathrm{s}, 4 \mathrm{H}, \mathrm{CH}=\mathrm{C} . \mathrm{G} 1) .6 .56(\mathrm{~s}, 4 \mathrm{H}, \mathrm{C}=\mathrm{C} . \mathrm{G} 2) .6 .57 \sim 7.43(\mathrm{~m}$. 150H. Pl. G0 G3) ${ }^{13} \mathrm{C}-\mathrm{NMR}$ (ppm. $\mathrm{CDCl}_{3}$ ): $\tilde{o}-2.42$ (SiMe. G0), -0.16 (SiMe, G1). -3.29 (SiMe, G2). -0.92 (SiMe, G3). $142.90 .14+.58(\mathrm{C}=\mathrm{C} . \mathrm{G} 0) .1+3.04,163.65(\mathrm{C}=\mathrm{C} . \mathrm{Gl}) .1+5.40$. $158.31(\mathrm{C}=\mathrm{C}, \mathrm{G} 2), 89.25,107.48(\mathrm{C} \equiv \mathrm{C}), 127.59,122.71$. 128.45. 132.08 (Ph, G3). 125.29, 125.99. 126.40, 127.71. 128.08, 128.18, 129.04, 129.18, 158.83 (Ph G0 G2). Anal.

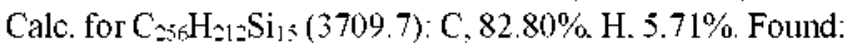
C. $82.43 \%$ H. $5.92 \%$. MALDI-TOF mass: $3723(\mathrm{M}+\mathrm{Na})^{+}$. UV/ Vis, $\lambda_{\max } 253 \mathrm{num}, \varepsilon_{\max } 2.12 \times 10^{5}$. GPC: PDI value $\left(\mathrm{M}_{*} / \mathrm{M}_{i}\right), 1.03$ (3166/3069); Rt, $17.25 \mathrm{~min}$.

4G[2,2,2,2,2]-32PA: $3.00 \mathrm{~g}(0.5+\mathrm{mmol})$ of $4 \mathrm{G}[2.2,2.2 .2]-$
$32 \mathrm{Cl}$ and $20 \mathrm{~mL}$ ( $20 \mathrm{mmol}$ ) of lithium phenylacetylide were used by the same procedure as the above for IG[2,0.2]-4PA. The mixture refluxed for $10 \mathrm{hrs}$. Yields: $3.13 \mathrm{~g}(76 \% .0 .4 \mathrm{l}$ numol) as yellow glassy material. ${ }^{1} \mathrm{H}-\mathrm{NMR}$ (ppm. $\mathrm{CDCl}_{3}$ ): $\delta$ $-0.88,-0.84,-0.83$ (s. $48 \mathrm{H}, \mathrm{SiMe}, \mathrm{G} 0 \sim \mathrm{G} 3$ ), $0.3 \mathrm{l}$ (s, $48 \mathrm{H}$, SiMe. G4), $5.80 \sim 6.98,6.46 \sim 6.60(30 \mathrm{H} . \mathrm{CH}=\mathrm{C}$. G0 G3), $6.60 \sim 6.76,6.76 \sim 7.62(\mathrm{~m}, 310 \mathrm{H}, \mathrm{Ph}, \mathrm{G} 0 \sim \mathrm{GH})$ ) ${ }^{13} \mathrm{C}-\mathrm{NMR}$ (ppm, $\mathrm{CDCl}_{2}$ ): ồ -3.26 (SiMe, G(1-G2), -1.10 (SiMe. G3). -0.80 (SiMe. G4). 89.16. 107.48 (C=C). 142.82. 144.49, 145.34, $158.13(\mathrm{C}=\mathrm{C}, \mathrm{G} 0 \sim \mathrm{G} 3), 122.58,126.30,127.64,128.04$. 128.95. 131.80, 132.19.163.65 (Pl, G( -G2). Anal. Calc. for $\mathrm{C}_{528} \mathrm{H}_{436} \mathrm{Si}_{31}(7.640):$ C. $82.93 \%$. H. 5.70\%. Found: C. $81.99 \%$. H. 5.79\%. MALDI- TOF mass: $767+63(\mathrm{M}+\mathrm{Na})^{-}$. UV/Nis, $\lambda_{\max } 253 \mathrm{~nm} . \varepsilon_{m a x} 4.47 \times 10^{5}$. GPC: PDI $\left(\mathrm{M}_{w} / \mathrm{M}_{n}\right) .1 .04(5.292 /$ 5.075): Rt. $16.35 \mathrm{~min}$.

$\mathbf{5 G}[2,2,2,2,2,2]-6+$ PA: $3.0 \mathrm{~g}(0.26$ nmol $)$ of $5 \mathrm{G}[2,2,2.2,2]-$ $64 \mathrm{Cl}$ and $20 \mathrm{~mL}$ (20 mmol) of lithium phenylacetylide were used by the same procedure as the above for $1 \mathrm{G}[2.0 .2]-4 \mathrm{PA}$. The mixture refluxed for 10 hrs. Yield: $3.22 \mathrm{~g} \mathrm{(79 \% .} 0.20$ numol) as yellow glassy material. ${ }^{1} \mathrm{H}-\mathrm{NMR}$ (ppm. $\mathrm{CDCl}_{2}$ ): $\delta$ $-1.06 \sim-0.64(96 \mathrm{H}$. SiMe, G0 G4), 0.16-0.48 (96H, SiMe, G5). $5.78 \sim 6.02 .6 .48 \sim 6.58(62 \mathrm{H}, \mathrm{CH}=\mathrm{C} . \mathrm{G} 0 \sim \mathrm{G} 5) .6 .48 \sim 6.76$. $6.76-7.6+(630 \mathrm{H}, \mathrm{Ph}, \mathrm{G} 0-\mathrm{G} 5) .{ }^{12} \mathrm{C}-\mathrm{NMR}\left(\mathrm{ppm} . \mathrm{CDCl}_{3}\right): \delta$ -3.21 (SiMe. G0 G4), -0.90 (SiMe. G5). 89.30. 107.51 (C $\equiv C$ ). 142.85. 144.52, 145.34. $158.13(\mathrm{C}=\mathrm{C}, \mathrm{G} 0-\mathrm{G} 4) .122 .65$. 126.39. 127.60. 128.21, 128.85. 132.05. 132.19. 164.23 (Ph. G0 G2). Anal. Calc. for $\mathrm{C}_{10 ; 2} \mathrm{H}_{88+} \mathrm{Si}_{63}$ (15.512): C. $82.93 \%$, H. $5.70 \%$. Found: C. $82.65 \%$. H, 5.43\% UV/Vis. $\lambda_{\text {max }} 253 \mathrm{~nm}$, $\varepsilon_{\max } 8.16 \times 10^{5}$. GPC: PDI $\left(\mathrm{M}_{11} / \mathrm{M}_{13}\right), 1.04(11.226 / 10.709): \mathrm{Rt}$. 16.02 nin.

$6 \mathrm{G}[2,2,2,2,2,2,2]-128 P A: 3.0 \mathrm{~g}(0.13 \mathrm{mmol})$ of $6 \mathrm{G}[2.2 .2$. $2.2 .2,2]-128 \mathrm{Cl}$ and $20 \mathrm{~mL}(20 \mathrm{mmol})$ of of lithium phenylacetylide were used by the same procedure as the above for IG $[2,0,2]-4 P A$. The mixture refluxed for 10 hrs. Yield: 3.00 $\mathrm{g}(74 \%, 0.095 \mathrm{mmol})$ as yellow glassy material. ${ }^{\mathrm{l}} \mathrm{H}-\mathrm{NMR}$ (ppm. $\mathrm{CDCl}_{3}$ ): $\delta-0.96 \sim-0.60(192 \mathrm{H}$. SiMe, $\mathrm{G} 0 \sim \mathrm{G} 5) .0 .20 \sim$ $0.49(192 \mathrm{H}$. SiMe, G6), 5.82 6.14 (126H, CH=C, G0^G5), $6.50 \sim 7.68\left(1270 \mathrm{H}, \mathrm{Ph}, \mathrm{G0}\right.$ G6) ${ }^{13} \mathrm{C}-\mathrm{NMR}\left(\mathrm{ppm} . \mathrm{CDCl}_{3}\right): \delta$ -3.12 ( $\mathrm{SiMe}, \mathrm{G} 0 n \mathrm{G5}$ ), -0.90 ( $\mathrm{SiMe}(\mathrm{G} 6)$ ), 89.34, 107.50 (C $\equiv \mathrm{C})$. $142.83,1+4.49,14+81,145.40 .158 .00(\mathrm{C}=\mathrm{C}, \mathrm{G}(0-\mathrm{G} 5), 122.63$, 126.49. 127.60. 128.05. 128.20. 128.83. 132.03. $163.38(\mathrm{Ph}$. G0-G6). Anal. Calc for $\mathrm{C}_{2161} \mathrm{H}_{1}: \mathrm{Si}_{12}=(31.256)$ : C, $82.93 \%$, H. $5.69 \%$. Found: C. $82.26 \%$. H. $5.69 \%$ UV/Vis. $\lambda_{\max } 253 \mathrm{~nm}$. $\varepsilon_{\max } 1.36 \times 10^{6}$. GPC: PDI $\left(\mathrm{M}_{11} / \mathrm{M}_{n}\right), 1.07(14,747 / 13,774)$ : Rt. 15.54 min.

$7 \mathrm{G}[2,2,2,2,2,2,2,2]-256 \mathrm{PA}: 1.00 \mathrm{~g}(0.022 \mathrm{mmol})$ of $7 \mathrm{G}[2$. $2.2 .2,2.2,2.2]-256 \mathrm{Cl}$ and $7 \mathrm{~nL}$ ( $7 \mathrm{mmol}$ ) of of lithium phenylacetylide were used by the same procedure as the above for $1 \mathrm{G}[2,0.2]-4 \mathrm{PA}$. The mixture refluxed for 10 lurs. Yield: $1.08 \mathrm{~g}$ $\left(82 \%, 0.017 \mathrm{mmol}\right.$ ) as yellow glassy material. ${ }^{\mathrm{H}} \mathrm{H}-\mathrm{NMR}$ (ppm. $\left.\mathrm{CDCl}_{3}\right): \delta-1.05 \sim-0.70(384 \mathrm{H}, \mathrm{SiMe}, \mathrm{G} 0 \sim \mathrm{G} 6) .0 .02 \sim 0.50$ (38+H. SiMe. G7), 5.78 -6.02 (25+H. CH=C. G0 -G6). 6.29 $7.60(\mathrm{~m}, 2550 \mathrm{H}, \mathrm{Pl}, \mathrm{G} 0 \sim \mathrm{G} 5) .{ }^{13} \mathrm{C}-\mathrm{NMR}\left(\mathrm{ppm}, \mathrm{CDCl}_{3}\right): \hat{\delta}-4.60 \sim$

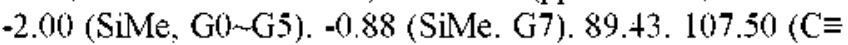
C) $142.79,144.50,145.34,158.26(\mathrm{C}=\mathrm{C}, \mathrm{G} 0 \sim \mathrm{G} 6), 122.64$. 126.39. 128.21. 129.03, 132.02, 163.43 (Pl. G0^G7). Anal. Calc. for $\mathrm{C}_{436} \mathrm{H}_{35: 2} \mathrm{Si}_{255}(62,744)$ : C. $82.93 \%$, H. $5.69 \%$. Found: C. $82.04 \%$, H, $5.82 \%$. UV/Vis. $\lambda_{\max } 253 \mathrm{~mm}, \varepsilon_{\max } 2.32$ 
$\times 10^{6}$. GPC: PDI value $\left(\mathrm{M}_{w} / \mathrm{M}_{1}\right) .1 .05(17,109 / 16.165)$; Rt. $15.15 \mathrm{~min}$.

(2) Preparation and characterization of the dendrimess with anthracene group on the periphery $\left(\mathrm{nG}\left[2,2^{n \cdot 1}, 2\right]-2^{n+1} \mathrm{AN}\right)$.

$1 \mathrm{G}[2,0,2]-4 \mathrm{AN}: 0.57 \mathrm{~g}(1.16 \mathrm{mmol})$ of $1 \mathrm{G}[2.0 .2]-4 \mathrm{Cl}$ dissolved in $25 \mathrm{~mL}$ of toluene was slowly added to the solution of $1.07 \mathrm{~g}(5.13 \mathrm{mmol})$ anthracene carbinol and $2.5 \mathrm{~g}$ ( $21.5 \mathrm{mmol}$ ) of TMEDA in $50 \mathrm{~mL}$ of dried toluene. Then, the reaction mixture was warmed to $60{ }^{\circ} \mathrm{C}$ for $30 \mathrm{~min}$. The TMEDAHCl salt was filtered off from the mixture. $1 \mathrm{G}[2.0 .2]-$ 4 AN was purified by silica gel chromatography with mixture of chloroform and hexane (2 to 1 ) removing the excess anthracene. Yield: $1.32 \mathrm{~g}(87 \%, 1.10 \mathrm{mmol})$ as white glassy material. ${ }^{1} \mathrm{H}$ NMR (ppm. $\mathrm{CDCl}_{3}$ ): 0 - -0.45 (S. 6H. SiMe. G0). 0.11 (s. $6 \mathrm{H}$. SiMe. Gl). $5.32 \sim 5.61$ (m. $8 \mathrm{H}, \mathrm{OCH}_{z}$ ). 6.50 (s. $2 \mathrm{H}$. $\mathrm{CH}=\mathrm{C}) .6 .88 \sim 7.20 .7 .20 \sim 7.35 .7 .82 \sim 8.02 .8 .02 \sim 8.26 .8 .26 \sim$ 8.44 ( $\mathrm{Ph}$, anthracene). ${ }^{13} \mathrm{CNMR}$ (ppm. $\mathrm{CDCl}_{3}$ ): $\delta-4.19$ (SiMe. G1), -0.46 (SiMe, G0), $57.43\left(\mathrm{OCH}_{2}\right), 124.51,124.77,125.79$. 126.23. 127.79, 127.91, 128.21, 128.78, 130.29, 130.69. 131.35. 143.93 (Ph. antluracene). 148.47, 159.52 ( $\mathrm{CH}=\mathrm{C}$ ). Anal. Calc. for $\mathrm{C}_{81} \mathrm{H}_{68} \mathrm{O}_{4} \mathrm{Si}_{3}(1177.67)$ : C. $81.59 \%$. H. $5.82 \%$. Found: $\mathrm{C}$. $81.25 \%$. H. $5.82 \%$. UV/Vis $\lambda_{\max } 258 \mathrm{~nm}$. $\varepsilon_{\max } 0.16 \times 10^{6}$. GPC: PDI $\left(\mathrm{M}_{w} / \mathrm{M}_{n}\right), 1.00(783 / 775)$; Rt, $19.32 \mathrm{~min}$.

2G[2,2,2]-8AN: $0.85 \mathrm{~g}(0.70 \mathrm{mmol})$ of $2 \mathrm{G}[2.2 .2]-8 \mathrm{Cl}$ and $1.0 \mathrm{~g}(8.6 \mathrm{mmol})$ of TMEDA and $1.40 \mathrm{~g}(6.72 \mathrm{mmol})$ of anthracene carbinol were used by the same procedure as the above for $1 \mathrm{G}[2.0 .2]-4 \mathrm{AN}$. Yield: $1.68 \mathrm{~g}(93 \%, 0.65 \mathrm{mmol})$ as light yellow to white glassy material. ${ }^{~} \mathrm{H} \mathrm{NMR}$ (ppm. $\mathrm{CDCl}_{3}$ ): ó -0.56 (s. 6H. SiMe. G0). -0.48 (s. 6H. SiMe. Gl) -0.00 (s. $12 \mathrm{H}$, SiMe, G2), $5.09-5.52\left(\mathrm{~m} .16 \mathrm{H} . \mathrm{OCH}_{2}\right), 6.21(\mathrm{~s}, 2 \mathrm{H}$. $\mathrm{CH}=\mathrm{C} . \mathrm{Gl}), 6.59$ (s. $+\mathrm{H} . \mathrm{CH}=\mathrm{C}(\mathrm{G} 2), 6.69 \sim 6.82,6.82 \sim 6.96$. 6.96 7.13. 7.13 7.51. 7.75 7.93. 7.93 8.20.8.20 8.45 (Ph. antluracene). ${ }^{13} \mathrm{C}$ NMR (ppm. $\mathrm{CDCl}_{3}$ ): $\delta-4.16$ (SiMe, G2). -2.62 (SiMe. G0)), -0.25 (SiMe. Gl)), $57.34\left(\mathrm{OCH}_{2}\right), 124.46$. $124.77,125.72,125.89,126.37,127.49,127.58,127.77$. 127.84. 128.70, 130.23.130.66, 131.31. 143.71 (Ph. anthracene), 145.01. 145.66. 160.65, $163.60(\mathrm{CH}=\mathrm{C})$. Anal Calc. for $\mathrm{C}_{1 \% 6} \mathrm{H}_{1+8} \mathrm{O}_{8} \mathrm{Si}$ ( $(2587.70)$ : C. $81.69 \%$. H. $5.76 \%$. Found: $\mathrm{C}$. $82.38 \%$. H. $5.72 \%$. UV/Vis. $\lambda_{\max } 258 \mathrm{~nm}$. $\varepsilon_{\max } 0.40 \times 10^{6}$. GPC: PDI value $\left(\mathrm{M}_{w} / \mathrm{M}_{n}\right), 1.02$ (1655/1612): Rt, $18.30 \mathrm{~min}$.

3G[2,2,2,2]-16AN: $0.61 \mathrm{~g}(0.23 \mathrm{mmol})$ of $3 \mathrm{G}[2.2 .2 .2]-$ $16 \mathrm{Cl}$ and $1.2 \mathrm{~g}(10.3 \mathrm{mmol})$ of TMEDA and $0.92 \mathrm{~g}(4.42$ mmol) of anthracene carbinol were used by the same procedure as the above for $1 \mathrm{G}[2.0,2]-4 \mathrm{AN}$. Yield: $1.20 \mathrm{~g}(97 \%$. $0.22 \mathrm{mmol}$ ) as yellow glassy material. ${ }^{1} \mathrm{H}-\mathrm{NMR}$ (ppm.

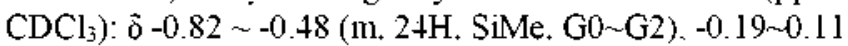
(m. 24H. SiMe.G3), 5.025.48 (m, 32H, OCH $), 6.01$ (s, $2 \mathrm{H}$. $\mathrm{CH}=\mathrm{C} . \mathrm{GIP}$ ) 6.18 (s. $+\mathrm{H} . \mathrm{CH}=\mathrm{C} . \mathrm{G} 2), 6.50$ (s. $8 \mathrm{H} . \mathrm{CH}=\mathrm{C}$. G3). $6.60 \sim 6.91,6.91 \sim 7.12 .7 .12 \sim 7.50,7.67 \sim 7.90 .7 .90 \sim 8.17$, $8.17 \sim 8.46$ (Ph. anthracene). ${ }^{13} \mathrm{C}-\mathrm{NMR}$ (ppm. $\mathrm{CDCl}_{3}$ ): 0 - -4.17 (SiMe (G3)). -2.77 (SiMe (G2)). -2.29 (SiMe (G1)) - -0.16 (SiMe $(\mathrm{G} 0)), 57.30\left(\mathrm{OCH}_{2}\right), 124.46 .124 .69,125.69 .125 .73$, 125.84. 126.38, 127.51, 127.81, 128.68, 130.20, 130.65. 131.28. 142.91, 143.71, 145.11. 145.32 (Ph, antluacene). 144.77. $145.43,160.54,160.61,163.76,164.90(\mathrm{CH}=\mathrm{C})$. Anal. Calc. for $\mathrm{C}_{368} \mathrm{H}_{3128} \mathrm{O}_{16} \mathrm{Si}_{15}(\mathrm{Mw}: 5407.75)$ : $\mathrm{C}, 81.74 \%, \mathrm{H}$, $5.74 \%$. Found: C. $81.47 \%$. H, $5.72 \%$. UV/Vis. $\lambda_{\text {max }} 258 \mathrm{~mm}$. $\varepsilon_{\max } 0.83 \times 10^{6}$. GPC: PDI value $\left(\mathrm{M}_{w} / \mathrm{M}_{n}\right), 1.04(5.1+3 / 4.935)$ :

\section{Rt, 17.16 min}

$4 \mathrm{G}[2,2,2,2,2]-32 \mathrm{AN}: 0.85 \mathrm{~g}(0.16 \mathrm{mmol})$ of $4 \mathrm{G}[2,2,2,2,2]-$ $32 \mathrm{Cl}$ and $0.8 \mathrm{~g}(6.8 \mathrm{mmol})$ of TMEDA and $1.29 \mathrm{~g}(6.18 \mathrm{mmol})$ of anthracene carbinol were used by the same procedure as the above for $\mathrm{IG}[2.0 .2]-4 \mathrm{AN}$. Yield: $1.62 \mathrm{~g}(92 \%, 0.15 \mathrm{mmol})$ as light yellow glassy material. ${ }^{l} \mathrm{H}-\mathrm{NMR}$ (ppm. $\mathrm{CDCl}_{3}$ ): $\delta-0.80$ $\sim-0.40(\mathrm{~m}, 48 \mathrm{H}$. SiMe),$-0.19 \sim 0.12(\mathrm{~m}, 48 \mathrm{H}, \mathrm{SiMe}) .5 .00 \sim$ 5.52 (n. $64 \mathrm{H} . \mathrm{OCH}_{2}$ ), 6.01 (s. $2 \mathrm{H}, \mathrm{CH}=\mathrm{C} . \mathrm{Gl}$ ), 6.09 (s. $4 \mathrm{H}$, $\mathrm{CH}=\mathrm{C}, \mathrm{G} 2), 6.15(\mathrm{~s}, 8 \mathrm{H}, \mathrm{CH}=\mathrm{C} . \mathrm{G} 3 \mathrm{P}), 6.50(\mathrm{~s}, 16 \mathrm{H} . \mathrm{CH}=\mathrm{C}$, G+P), 6.61 6.92. 6.92 7.08. 7.08 7.59. 7.59 7.87.7.87 8.09. 8.09 8.46 (Pl. antluracene). ${ }^{13} \mathrm{C}-\mathrm{NMR}$ (ppm. $\mathrm{CDCl}_{3}$ ): ô -4.18 (SiMe, G4), -2.75 (SiMe, G3). -2.58 (\$iMe, G2), -2.34 ( SiMe. Gl). -0.27 (SiMe, G0). $57.26\left(\mathrm{OCH}_{2}\right), 124.41,124.63$, 125.64. 126.08, 126.37, 127.77, 128.62, 130.14, 130.60 . 131.21. 142.33. 143.63 ( $\mathrm{Ph}$ anthracene). 144.81. 145.27. 160.60. 164.64. 164.79. 165.78 (CH=C). Anal. Calc. for $\mathrm{C}_{752} \mathrm{H}_{628} \mathrm{O}_{32} \mathrm{Si}_{21}(\mathrm{Mw}: 11,047)$ : C. 81.76\%, H. 5.73\%. Found: C. $81.78 \%, \mathrm{H}, 5.71 \%$. UV/Vis. $\lambda_{\text {max }} 258 \mathrm{~nm} . \varepsilon_{\text {mar }} 1.67 \times 10^{\circ}$. GPC: PDI value $\left(\mathrm{M}_{w} / \mathrm{M}_{n}\right) .1 .01(9.843 / 9.743):$ Rt. $16.54 \mathrm{~min}$.

$\mathbf{5 G}[2,2,2,2,2,2]-64 \mathrm{AN}: 0.57 \mathrm{~g}(0.050 \mathrm{mmol})$ of $5 \mathrm{G}[2.2,2.2$. $2.2]-64 \mathrm{Cl}$ and $0.41 \mathrm{~g}$ of TMEDA $(3.56 \mathrm{mmol})$ and $0.74 \mathrm{~g}$ (3.56 mmol) of anthracene carbinol were used by the same procedure as the above for $1 \mathrm{G}[2,0,2]-4 \mathrm{AN}$. Yield: $1.01 \mathrm{~g}$ $(91 \% .0 .045 \mathrm{mmol})$ as light yellow glassy material. ${ }^{1} \mathrm{H}-\mathrm{NMR}$ (ppm, $\left.\mathrm{CDCl}_{3}\right):-0.84 \sim-0.59(96 \mathrm{H}, \mathrm{SiMe}, \mathrm{G} 5),-0.32 \sim-0.08$ $(96 \mathrm{H}$. SiMe, GOnG4). 4.74 5.19(128H, OCH, G5). $5.84 \sim 6.19$ $(62 \mathrm{H}, \mathrm{CH}=\mathrm{C}, \mathrm{G} 0 \sim \mathrm{G} 4), 6.32 \sim 6.48,6.48 \sim 7.36,7.36 \sim 7.60$, $7.60 \sim 7.82 .7 .82 \sim 8.12$ (n. $886 \mathrm{H}, \mathrm{Ph}$, antluacene ). ${ }^{12} \mathrm{C}-\mathrm{NMR}$ (ppm, $\mathrm{CDCl}_{3}$ ): $\tilde{o}-4.82$ (SiMe.G5), -2.79 (SiMe, G0 G4). $57.61 \quad\left(\mathrm{OCH}_{2}\right) .122 .23$. 123.14. 124.66. 125.30. 127.25. 127.80, 128.23, 128.69, 129.04, 130.22, 131.28, 133.54 . 134.13. 137.88. $138.61,143.17$ ( $\mathrm{Ph}$, anthracene). 144.18 , $159.88(\mathrm{CH}=\mathrm{C})$. Anal.Calc. for $\mathrm{C}_{15} \mathrm{H}_{1268} \mathrm{O}_{64} \mathrm{Si}_{53}(\mathrm{Mw}$ : 22.296): C. $81.80 \%$. H. $5.68 \%$. Found: C. $81.52 \%$. H. $5.66 \%$. UVNis, $\lambda_{\text {mar }} 258 \mathrm{~nm}, \varepsilon_{\text {mar }} 2.99 \times 10^{6}$. GPC: PDI value $\left(\mathrm{M}_{1 \mathrm{r}} / \mathrm{M}_{n}\right) .1 .03$ (12.675/12.295): Rt. $15.97 \mathrm{~min}$.

$6 \mathrm{G}[2,2,2,2,2,2,2]-128 \mathrm{AN}: 0.58 \mathrm{~g}(0.025 \mathrm{mmol})$ of $6 \mathrm{G}[2.2$, $2.2 .2 .2 .2]-128 \mathrm{Cl}$ and $0.4 \mathrm{~g}$ of TMEDA $(3.56 \mathrm{mmol})$ and 0.74 $\mathrm{g}(3.56 \mathrm{mmol})$ of anthracene carbinol were used by the same procedure as the above for $1 \mathrm{G}[2,0,2]-4 \mathrm{AN}$. Yield: $0.99 \mathrm{~g}$ $(88 \% .0 .022 \mathrm{mmol})$ as light yellow glassy material. ${ }^{1} \mathrm{H}-\mathrm{NMR}$ (ppm, $\left.\mathrm{CDCl}_{3}\right):$ ô $-0.94 \sim-0.56(192 \mathrm{H}$. SiMe, G6)), $-0.58 \sim$

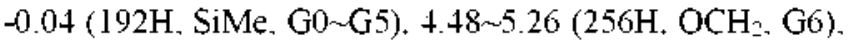
$5.84 \sim 6.19(126 \mathrm{H}$. $\mathrm{CH}=\mathrm{C}, \mathrm{GO} \sim \mathrm{G} 5) .6 .22 \sim 7.32 .7 .32 \sim 7.60$, $7.60 \sim 7.82 .7 .82 \sim 8.12\left(\mathrm{~m}, 1782 \mathrm{H}, \mathrm{Ph}\right.$, anthracene). ${ }^{13} \mathrm{C}-\mathrm{NMR}$ (ppm. $\mathrm{CDCl}_{2}$ ): $\delta$-4.87 (SiMe. G6). -2.79 (SiMe. G0 G5). $60.48\left(\mathrm{OCH}_{2}\right) .121 .83,123.14 .123 .62 .124 .78,127.25 .127 .80$. $128.70 .130 .26,131.36 .133 .55 .134 .15 .138 .60 .143 .00(\mathrm{Ph}$. anthracene). $144.18,159.88(\mathrm{CH}=\mathrm{C})$. Anal.Calc. for $\mathrm{C}_{31566^{-}}$ $\mathrm{H}_{2.48} \mathrm{O}_{1.2 \mathrm{Si}} \mathrm{Si}_{12}(\mathrm{Mw} .44 .824):$ C. $81.81 \%$. H. 5.68\%. Found: $\mathrm{C}$. $81.42 \%$. H. $5.64 \%$. UV/Vis. $\lambda_{\max } 258 \mathrm{~nm}$. $\varepsilon_{\max } 5.80 \times 10^{6}$ GPC: PDI value $\left(\mathrm{M}_{w} / \mathrm{M}_{n}\right) .1 .05(15.315 / 14.474)$ : Rt. 15.45 min.

$7 \mathrm{G}[2,2,2,2,2,2,2,2]-256 \mathrm{AN}: 0.44 \mathrm{~g}(0.095 \mathrm{mmol})$ of $7 \mathrm{G}[2$, $2.2 .2 .2 .2 .2 .2]-256 \mathrm{Cl}$ and $0.31 \mathrm{~g}$ of TMEDA (2.69 $\mathrm{mmol})$ and $0.56 \mathrm{~g}(2.69 \mathrm{mmol})$ of antluracene carbinol were used by the same procedure as the above for $1 \mathrm{G}[2,0.2]-4 \mathrm{AN}$. Yield: 0.75 $\mathrm{g}(88 \%, 0.083 \mathrm{mmol})$ as light yellow glassy material. ${ }^{1} \mathrm{H}-\mathrm{NMR}$ 
(ppm, CDCl $)$ ) ồ $-0.98 \sim 0.50(38+\mathrm{H}$. SiMe, G7)), $-0.34 \sim 0.24$ $(38+\mathrm{H}$. SiMe, G0 G6). 4.88 5.34 (512H, OCH, $\mathrm{G} 7), 6.00 \sim$ $6.32(254 \mathrm{H} . \mathrm{CH}=\mathrm{C} . \mathrm{GO} \sim \mathrm{G} 6), 6.32 \sim 7.36 .7 .52 \sim 7.80 .7 .80 \sim 8.00$. $8.00 \sim 8.2+\left(\mathrm{m}, 3574 \mathrm{H}, \mathrm{Ph}\right.$ and anthracene). ${ }^{13} \mathrm{C}-\mathrm{NMR}$ (ppm. $\mathrm{CDCl}_{3}$ ): $\delta-4.82$ (SiMe. G7). -2.31 (SiMe. G0 G6). 60.25 $\left(\mathrm{OCH}_{2}\right) .122 .21,123.26,127.24$. 127.32. 127.54, 128.67. 130.34. 131.47, 131.85, 134.14, 138.75, 143.00 (Plh anthracene). 144.00. $163.67(\mathrm{CH}=\mathrm{C})$. Antal. Calc. for $\mathrm{C}_{6128} \mathrm{H}_{5168} \mathrm{O}_{256} \mathrm{Si}_{555}$ (Mw: 89,880): C, $81.81 \%$, H. 5.68\%. Found: C, $81.39 \%$. H. $5.59 \%$. UV/Vis $\hat{t}_{\max } 258 \mathrm{~nm}$. $\varepsilon_{\max } 9.79 \times 10^{6}$. GPC: PDI $\left(\mathrm{M}_{w} / \mathrm{M}_{i}\right), 1.06(17.008 / 16.009)$; Rt. $15.30 \mathrm{~min}$.

(3) Preparation and characterization of the dendrimers with benzoxazole groups on the periphery (nG[2,2 $\left.2^{\mathrm{n} \cdot 1}, 1\right]-2^{\mathrm{n}} \mathrm{BO}$ ).

1G[2,0,1]-2BO: $0.30 \mathrm{~g}(0.67 \mathrm{mmol})$ of $\mathrm{IG}[2.0 .1]-2 \mathrm{Cl}$ in 25 $\mathrm{ml}$ of toluene slowly added to the mixed solution of $0.31 \mathrm{~g}$ (1.47 mmol) 2-(2-hydroxyphenyl)benzoxazole and $0.17 \mathrm{~g}$ (1.45 mmol) of TMEDA in $50 \mathrm{~mL}$ of dried toluene at room temperature. Then the reaction mixture was warned and kept at $60{ }^{\circ} \mathrm{C}$ for $1.5 \mathrm{hrs}$. The reaction mixture was filtered to remove TMEDA-HCl salt. $1 \mathrm{G}[2.0,1]-2 \mathrm{BO}$ was purified and isolated by silica gel chromatography of chloroform and hexane ( 3 to 2$)$. Yield: $0.41 \mathrm{~g}(77 \%, 0.51 \mathrm{mmol})$ as yellow glassy material. ${ }^{l} \mathrm{H}-\mathrm{NMR}$ (ppm. $\mathrm{CDCl}_{3}$ ): $\bar{o}-0.47(5,6 \mathrm{H}$. SiMe. G0), 0.29 (s. 12H. SiMe. Gl), 6.36 (s. $2 \mathrm{H} . \mathrm{CH}=\mathrm{C}$ ), $6.80 \sim 6.98$. $6.98 \sim 7.22 .7 .22 \sim 7.42 .7 .42 \sim 7.55 .7 .70 \sim 7.80,8.04 \sim 8.18$ ( $12 \mathrm{H}, \mathrm{BO} . \mathrm{Ph}$ ). ${ }^{13} \mathrm{C}-\mathrm{NMR}$ (ppm. CDCl $)$ ) ò -0.75 (SiMe. Gl). -0.29 (SiMe. G0). 110.62, 117.37, 119.21, 119.54. 124.96. 125.34 . 125.95, 127.07, 127.26, 127.85, 133.52, 139.97 . 144.97. 149.09, 158.66, 162.83, 164.05 (Ph. BO). Anal. Calc. for $\mathrm{C}_{48} \mathrm{H}_{46} \mathrm{~N}_{2} \mathrm{O}_{4} \mathrm{Si}_{3}(\mathrm{Mw}: 799.16)$ : C, 72.07\%, H, 5.75\%. N. $3.50 \%$. Found: C. $71.86 \%$. H. $5.57 \%$. N. $3.48 \%$. MALDI-TOF mass: $799.36(\mathrm{M})$. UV/Vis, $\lambda_{\max } 293 \mathrm{~nm} . \varepsilon_{\operatorname{mix}} 0.21 \times 10^{5}$. GPC: PDI $\left(\mathrm{M}_{w} / \mathrm{M}_{n}\right) .1 .01$ (682/674): Rt. $19.93 \mathrm{~min}$.

2G[2,2,1]-4BO: $0.45 \mathrm{~g}(0.40 \mathrm{mmol})$ of $2 \mathrm{G}[2.2 .1]-4 \mathrm{Cl}$ and $0.20 \mathrm{~g}$ of TMEDA $(1.17 \mathrm{mmol})$ and $0.39 \mathrm{~g}(1.71 \mathrm{mmol})$ of 2-(2-hydroxyphenyl)benzoxazole were used by the same procedure as the above for $1 \mathrm{G}[2.0 .1]-2 \mathrm{BO}$. Yield: $0.56 \mathrm{~g}(76 \%$, $0.30 \mathrm{mmol}$ ) as light yellow glassy materials. ${ }^{\mathrm{H}} \mathrm{H}-\mathrm{NMR}$ (ppm. $\mathrm{CDCl}_{3}$ ): $\delta-0.7 \mathrm{l}$ (s.6H. SiMe. G0), -0.53 (s, 6H, SiMe. Gl). 0.22 (s. 24H, SiMe, G2), 6.04 (s, 12H, CH=C, G0). 6.31 (s. $\mathrm{I} 2 \mathrm{H}, \mathrm{CH}=\mathrm{C}, \mathrm{Gl}), 6.65 \sim 6.87,6.98 \sim 7.15,7.15 \sim 7.35,7.35 \sim$ 7.50. 7.68 7.79.8.04 8.12 (6+H. BO. Ph). ${ }^{13} \mathrm{C}-\mathrm{NMR}$ (ppm. $\mathrm{CDCl}_{3}$ ): $\delta-0.50$ (SiMe. G2)) -0.29 (SiMe. G0 Gl), 110.87. $117.64 .119 .77,125.21,125.58 .126 .14,127.45 .127 .57 .127 .63$. 127.97. 133.77. 140.24. 144.46. 149.36. 158.95. 163.12 (Pl. $\mathrm{BO}$ ). Anal. Calc. for $\mathrm{C}_{11} \mathrm{H}_{1}{ }_{14} \mathrm{~N}_{4} \mathrm{O}_{8} \mathrm{Si}_{-}(\mathrm{Mw}: 1.830)$ : C. $73.44 \%$. H. $5.68 \%$, N. $3.06 \%$. Found: C. $72.90 \%$, H. $5.73 \%$. N. $3.42 \%$. UV/Vis $\lambda_{\max } 293 \mathrm{~nm} . \varepsilon_{\max } 0.46 \times 10^{5}$. GPC: PDI value $\left(\mathrm{M}_{\mathrm{r}} /\right.$ $\left.\mathrm{M}_{s}\right), 1.02(1.110 / 1.082)$ : Rt. $18.92 \mathrm{~min}$.

3G[2,2,2,1]-8BO: $0.49 \mathrm{~g}(0.19 \mathrm{mmol})$ of $3 \mathrm{G}[2,2.2,1]-8 \mathrm{Cl}$ and $0.20 \mathrm{~g}$ of TMEDA $(1.17 \mathrm{mmol})$ and $0.37 \mathrm{~g}(1.75 \mathrm{mmol})$ of 2-(2-hydroxyphenyl)benzoxazole were used by the same procedure as the above for $\mathrm{IG}[2,0.1]-2 \mathrm{BO}$. Yield: $0.58 \mathrm{~g}(78 \%$. $0.15 \mathrm{mmol}$ ) as light yellow glassy material. ${ }^{\mathrm{H}} \mathrm{H}-\mathrm{NMR}$ (ppm. $\mathrm{CDCl}_{3}$ ): $\delta-0.80$ (s. 12H. SiMe. G2) $-0.5 \mathrm{l}$ (s. 6H. SiMe. Gl). $0.16(\mathrm{~s}, 48 \mathrm{H}, \mathrm{SiMe}, \mathrm{G} 3), 6.07$ ( $5,6 \mathrm{H} . \mathrm{CH}=\mathrm{C}, \mathrm{G} 0 \sim \mathrm{Gl}), 6.22$ (s. $8 \mathrm{H} . \mathrm{CH}=\mathrm{C} . \mathrm{G} 2), 6.58 \sim 6.87 .6 .95 \sim 7.14 .7 .14 \sim 7.37,7.37 \sim$ 7.48, 7.72 7.98,8.02 8.14 (134H. BO. Ph). ${ }^{13} \mathrm{C}-\mathrm{NMR}$ (ppm.
$\mathrm{CDCl}_{3}$ ): ồ -2.86 (SiMe, G(1-G1), -2.25 (SiMe. G2). -2.22 (SiMe (G3). 110.61, 117.35, 119.18, 119.50, 124.94, 125.31, $125.82,127.05,127.33,127.61,128.32,133.51,137.80,1+1.02$. $142.79 .14+.22 .14+78,149.05,158.66,162.82,163.74,164.88$ (Plh, BO). Anal. Calc. for $\mathrm{C}_{24} \mathrm{H}_{22}\left(\mathrm{~N}_{8} \mathrm{O}_{16} \mathrm{Si}_{15}(\mathrm{Mw}: 3.888): \mathrm{C}\right.$. $74.00 \%$. H. $5.65 \%$, N, $2.88 \%$. Found: C. $73.24 \%$. H. $5.88 \%$, N. $3.02 \%$. UV/Vis $\lambda_{\max } 293 \mathrm{~nm}, \varepsilon_{\max } 0.86 \times 10^{5}$. GPC: PDI value $\left(\mathrm{M}_{w} / \mathrm{M}_{n}\right), 1.02(3.472 / 3,389)$; Rt, $17.72 \mathrm{~min}$.

$+\mathrm{G}[2,2,2,2,1]-16 \mathrm{BO}: 0.32 \mathrm{~g}(0.061 \mathrm{mmol})$ of $4 \mathrm{G}[2,2.2,2$, 1]-16Cl and $0.12 \mathrm{~g}$ of TMEDA ( $1.17 \mathrm{mmol})$ and $0.12 \mathrm{~g} \mathrm{(1.07}$ numol) of 2-(2-hydroxyphenyl)benzoxazole were used by the same procedure as the above for $I G[2,0.1]-2 \mathrm{BO}$. Yield: 0.40 $\mathrm{g}\left(82 \%, 0.05\right.$ numol) as light yellow glassy material. ${ }^{\mathrm{H}} \mathrm{H}-\mathrm{NMR}$ (ppm. $\mathrm{CDCl}_{3}$ ): $\delta-0.89$ (s. 18H. SiMe. G0. G2). -0.81 (s. 12H. SiMe. G3), -0.57 (s. 24H. SiMe. Gl), 0.15 (s.96H. SiMe. G4). $5.94,5.97(1+\mathrm{H} . \mathrm{CH}=\mathrm{C}, \mathrm{G} 0 \sim \mathrm{G} 2), 6.18(\mathrm{~s}, 16 \mathrm{H} . \mathrm{CH}=\mathrm{C}, \mathrm{G} 3)$, $6.5+\sim 6.84,6.90 \sim 7.14 .7 .14 \sim 7.34 .7 .34 \sim 7.46,7.66 \sim 7.96,8.02 \sim$ $8.16(278 \mathrm{H}, \mathrm{BO}, \mathrm{Pl}) .{ }^{13} \mathrm{C}-\mathrm{NMR}$ (ppm. $\mathrm{CDCl}_{3}$ ): ô -2.87 (SiMe, G0〜G3). -0.85 (SiMe, G4). 110.61, 117.35, 119.18, 119.50 . 124.94. 125.31. 125.82, 127.05, 127.33. 127.61, 128.32, 133.51. 137.80. 141.02, $142.79,144.22 .144 .78,149.05$, 158.66. 162.82, 163.74, $164.88(\mathrm{Ph}, \mathrm{BO})$. Anal. Calc. for $\mathrm{C}_{496} \mathrm{H}_{45} \mathrm{~N}_{16} \mathrm{O}_{32} \mathrm{Si}_{31}$ (Mw: 8,008): C. $74.32 \%, \mathrm{H}, 5.64 \%, \mathrm{~N}$, $2.79 \%$. Found: C. $73.82 \%$. H. $5.73 \%$. N. $3.21 \%$ UV/Vis. $\lambda_{\max }$ $293 \mathrm{lnt}, \varepsilon_{\max } 1.77 \times 10^{5}$. GPC: PDI $\left(\mathrm{M}_{n} / \mathrm{M}_{n}\right) .1 .00(5,588 /$ 5.562): Rt. $16.84 \mathrm{~min}$.

5G[2,2,2,2,2,1]-32BO: $0.40 \mathrm{~g}(0.037 \mathrm{mmol})$ of $\mathrm{G} 5-32 \mathrm{Cl}$ and $0.1+\mathrm{g}$ of TMEDA $(1.19 \mathrm{mmol})$ and $0.25 \mathrm{~g}(1.19 \mathrm{~mm}$. $)$ of 2-(2-hydroxy phenyl)benzoxazole were used by the same procedure as the above for $1 \mathrm{G}[2.0,1]-2 \mathrm{BO}$. Yield: $0.40 \mathrm{~g}(82 \%$, $0.050 \mathrm{mmol}$ ) as yellow glassy material. ${ }^{1} \mathrm{H}-\mathrm{NMR}$ (ppm. $\left.\mathrm{CDCl}_{2}\right): \delta-0.96 \sim-0.68(96 \mathrm{H}$. SiMe. G(0-G4), $0.13(192 \mathrm{H}$, SiMe. G5). 5.79 -6.05. 6.12 6.26 (62H. $\mathrm{CH}=\mathrm{C}, \mathrm{G}(\sim-\mathrm{G} 4)$, $6.50 \sim 6.82,6.82 \sim 7.12 .7 .12 \sim 7.30 .7 .30 \sim 7.44 .7 .62 \sim 7.80 .8 .00 \sim$ $8.14\left(566 \mathrm{H}, \mathrm{BO}, \mathrm{Pl}\right.$ ). ${ }^{12} \mathrm{C}-\mathrm{NMR}$ (ppm. CDCl 3 ): ô -0.44 (SiMe (G5)), $\quad-0.29$ (SiMe (G0 G4)). 119.86. $117.64,119.46$. $119.77 .125 .21,125.58,126.117,127.331$. 127.56. 127.95. 133.77. 140.24. 144.46. 149.35. 158.95, 163.12 (Ph and BO). Anal. Calc. for $\mathrm{C}_{1(6) 3} \mathrm{H}_{916} \mathrm{~N}_{32} \mathrm{O}_{64} \mathrm{Si}_{62}(\mathrm{Mw}: 16.248)$ : C. $74.44 \%$. H. $5.63 \%$. N, $2.75 \%$. Found: C, $73.99 \%$. H, 5.80\%, N. 3. $12 \%$. UV/Vis. $\lambda_{\max } 293 \mathrm{~nm}, \varepsilon_{\max } 3.65 \times 10^{5}$. GPC: PDI value $\left(\mathrm{M}_{n} / \mathrm{M}_{n}\right) .1 .0+(11.302 / 10.803)$ : Rt. 15.95 min.

$6 \mathrm{G}[2,2,2,2,2,2,1]-64 \mathrm{BO}: 0.32 \mathrm{~g}(0.014 \mathrm{mmol})$ of $6 \mathrm{G}[2.2 .2$. $2.2 .2,1]-6+\mathrm{Cl}$ and $0.12 \mathrm{~g}$ of TMEDA $(1.06 \mathrm{mmol})$ and $0.22 \mathrm{~g}$ (1.06 mmol) of 2-(2-hydroxyphenyl)benzoxazole were used by the same procedure as the above for $I G[2,0.1]-2 B O$. Yield: $0.36 \mathrm{~g}\left(79 \%\right.$. $0.111 \mathrm{~mm}$ ol) as yellow glassy material. ${ }^{\mathrm{H}} \mathrm{H}-\mathrm{NMR}$ (ppm. $\left.\mathrm{CDCl}_{3}\right):$ ò $-0.96 \sim-0.68(192 \mathrm{H}$, SiMe. G0 G5), 0.13 $(38+\mathrm{H}$. SiMe. G6). $5.68-6.30$ (126H. $\mathrm{CH}=\mathrm{C}, \mathrm{G}(1-\mathrm{G} 5)$, $6.50 \sim 6.82,6.82 \sim 7.12,7.12 \sim 7.30,7.30 \sim 7.44 .7 .62 \sim 7.80,8.00 \sim$ 8.14 (1142H. BO Ph). ${ }^{3} \mathrm{C}-\mathrm{NMR}$ (ppm. CDCl $)$ ) $\tilde{\delta}-0.50$ (SiMe. G6). -0.29 (SiMe. G0-G5). 110.87, 117.64, 119.47. 119.77. 125.22 . 125.59, 127.33, 127.65, 128.44, 127.97, 128.442. $140.25,144.46 .149 .36,158.96 .163 .13$ (BO, Ph). Anal. Calc. for $\mathrm{C}_{2032} \mathrm{H}_{1 ; 8} \mathrm{~N}_{64} \mathrm{O}_{128} \mathrm{Si}_{127}$ (Mw: 32.632): C. $74.72 \%, \mathrm{H}$, $5.36 \%$, N, 2.74\%. Found: C. $73.91 \%$. H. $5.62 \%$, N, 3.04\%. UV/Nis, $\lambda_{\text {max }} 293 \mathrm{~nm}, \varepsilon_{\text {mar }} 7.08 \times 10^{5}$. GPC: PDI value $\left(\mathrm{M}_{1 /} / \mathrm{M}_{n}\right) .1 .06(12.538 / 11.775)$ : Rt. $15.53 \mathrm{~min}$. 
$7 \mathrm{G}[2,2,2,2,2,2,2,1]-128 \mathrm{BO}: 0.18 \mathrm{~g}(0.86 \mathrm{mmol})$ of $7 \mathrm{G}[2,2$, $2.2 .2 .2 .2 .1]-128 \mathrm{Cl}$ and $0.10 \mathrm{~g}$ of TMEDA $(0.86 \mathrm{mmol})$ and $0.18 \mathrm{~g}(0.86 \mathrm{mmol})$ of 2-(2-hy'droxyphenyl)benzoxazole were used by the same procedure as the above for $1 \mathrm{G}[2,0,1]-2 \mathrm{BO}$. Yield: $0.34 \mathrm{~g}(83 \% .0 .0051 \mathrm{mmol})$ as yellow glassy material. ${ }^{l} \mathrm{H}-\mathrm{NMR}\left(\mathrm{ppm}, \mathrm{CDCl}_{3}\right): \delta \cdot 1.02 \sim-0.62(38+\mathrm{H}, \mathrm{SiMe}, \mathrm{GO} \sim$ G6). $0.32 \sim 0.36(768 \mathrm{H}$. SiMe G7). 5.82 6.30 $(254 \mathrm{H} . \mathrm{CH}=\mathrm{C}$. G0 $\sim$ G6), 6.30 7.52. 7.60 7.80, 7.94 8.14 (229+H. BO, Ph). ${ }^{13} \mathrm{C}-\mathrm{NMR}$ (ppm, $\mathrm{CDCl}_{3}$ ): $\delta-2.39$ (SiMe, G0 G6), -0.61 (SiMe. G7). 117.43, 119.27, 119.58, 125.01. 125.39. 125.94, 127.13. $127.67 .133 .57,140.03,144.28 .149 .15,158.73,162.91$.

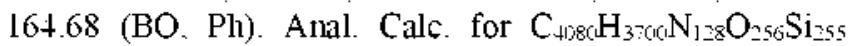
(Mw: 65.688): C. $74.53 \%$. H, 5.63\%, N. 2.72\%. Found: C. $73.86 \%$. H. $5.92 \%$. N. $3.02 \%$. UV/Vis. $\lambda_{\text {max }} 293$ nm. $\varepsilon_{\text {max }} 1.40$ $\times 10^{6} . \mathrm{GPC}$ : PDI $\left(\mathrm{M}_{w} / \mathrm{M}_{t}\right), 1.01(14.898 / 1+623)$ : Rt. 15.30 min.

\section{Results and Discussion}

Hydrosilation of bis(pheny lacety lenyl)dimethylsilane with two equivalent amounts of dichloromethylsilane gives bis(2.2-dichloromethylsilyl-2-phenylethenyl)dimethyl silane having four $\mathrm{Cl}-\mathrm{Si}$ bonds of two peripheral silyl groups. The coupling reaction of the four $\mathrm{Cl}-\mathrm{Si}$ bonds with lithium phenylacetylide introduces new four phenylacetylenyl groups into the core to give the $2^{\text {nd }}$ generation. Hy'drosilation of the four acetylene moieties with dichloromethylsilanes to give new $\mathrm{Cl}-\mathrm{Si}$ bonds and followed by the coupling reaction of the $\mathrm{Cl}-\mathrm{Si}$ bonds with lithium phenylacety lide are repetitively carried out to obtain the $3^{\text {nd }}$ to the $7^{\text {th }}$ generation having 256 pheny ethenyl groups $-[\mathrm{CH}=\mathrm{C}(\mathrm{Ph}) \mathrm{SiMe}]_{2}$ - in inner shells. Lastly instead of lithium phenylacetylide chromophores such as anthracene and benzoxazole are introduced by the coupling reaction of the terminal $\mathrm{Cl}-\mathrm{Si}$ bonds of each generation with hydroxyl groups of two chromophores.

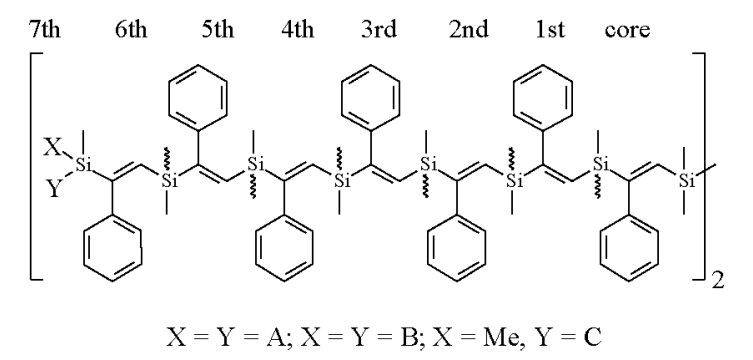

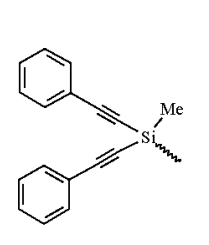

A

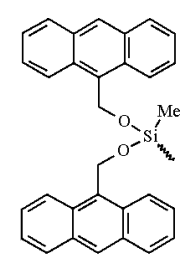

B

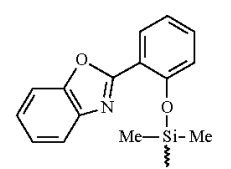

C
Scheme 1. The skeletal structures of pheny lethynylsilane dendrimers and peripheral functions on them. (A) Bis (phenvlethrnyl)methvlsilyl, (B)Bis (9-methyloxianthracenyl) methylsily: , (C) 2-Benzoxazolylphenyloxy dimethy lsilyl group)
Preparation of phenylethynylsilane dendrimers (nG[2,2 $2^{n \cdot 1}$, 2]-2 ${ }^{\mathrm{n}+1}$ PA; Type A). ${ }^{\text {sa. }}{ }^{\text {sb }}$ Cascade type synthetic method is applied to the preparation of phenylethy nylsilane dendrimers $\left(n G\left[2.2^{n \cdot l} \cdot 2\right]-2^{n-1}\right.$ PA. $\left.n=1 \sim 7\right)$. The first generation $(1 \mathrm{G}[2.0,2]-$ $4 \mathrm{Cl}$ ) with four growing points of $\mathrm{Cl}-\mathrm{Si}$ bonds has been prepared by the hydrosilation of bis(phenylacetlylenyl)dimethylsilane with two equivalent amount of dichloromethylsilane in the presence of dried platinum metal $(10 \%$ in active carbon). Sharp single peak from two protons of two double bonds is observed at $6.71 \mathrm{ppm}$ by ${ }^{1} \mathrm{H}$ NMR quantitatively. After removing catalyst from the mixture, is isolated the first generation of $\mathrm{IG}[2,0.2]-4 \mathrm{Cl}$ having air sensitive $\mathrm{Cl}-\mathrm{Si}$ bonds as light yellow material like gelatin in over $95 \%$. The $2^{\text {nd }}$ to the $7^{\text {th }}$ generations of $\mathrm{nG}\left[2.2^{12 \cdot 1} \cdot 2\right]-2^{\mathrm{n}-1} \mathrm{Cl}(\mathrm{n}=2 \sim 7)$ are prepared in high yield by the same method as the above. The $1^{\text {st }}$ generation of $1 \mathrm{G}[2,0.2]-4 \mathrm{PA}$ having four phenylacetylenyl groups. $\mathrm{Me}_{2} \mathrm{Si}\left[\mathrm{CH}=\mathrm{C}(\mathrm{Ph}) \mathrm{Si}(\mathrm{Me})(\mathrm{C} \equiv \mathrm{CPh})_{2}\right]_{2}$, is prepared by the reaction of $1 \mathrm{G}[2.0 .2]-4 \mathrm{Cl}$ with four equivalent of lithium phenylacetylide at room temperature. The $1^{\text {st }}$ generation of $1 G[2,0.2]-4 P A$ is isolated as light yellowish solid in over $90 \%$ yield. The $2^{\text {nd }}$ generation of $2 \mathrm{G}[2,2.2]-8 \mathrm{PA}$ having 8 phenylacethy'lenyl groups to the $7^{\text {th }}$ generation of $7 \mathrm{G}\left[2,2^{6}, 2\right]-256 \mathrm{PA}$ having 256 phenylacethylenyl groups are prepared by the same method as the above. The reaction rate of the hydrosilation becomes slower with increasing the generation from $\mathrm{IG}[2,0,2]-4 \mathrm{Cl}$ to $7 \mathrm{G}\left[2,2^{6}, 2\right]-256 \mathrm{Cl}$.

The dendrimers of $\mathrm{nG}\left[2,2^{\mathrm{n}-1}, 2\right]-2^{\mathrm{n}+\mathrm{j}} \mathrm{PA}(\mathrm{n}=1 \sim 7$ : Scheme 2) are quite stable in atmospheric condition, they are identified by ${ }^{1} \mathrm{H}$ and ${ }^{13} \mathrm{C}$ NMR. MALDI-MS. elemental analy sis. The purity of the respective dendrimer is confirmed by GPC, in which very narrow peak (polydisperse index value close to unit 1.00 ) is observed at shorter retention time according to increasing its generation (Fig. 2 and Table 1). Especially

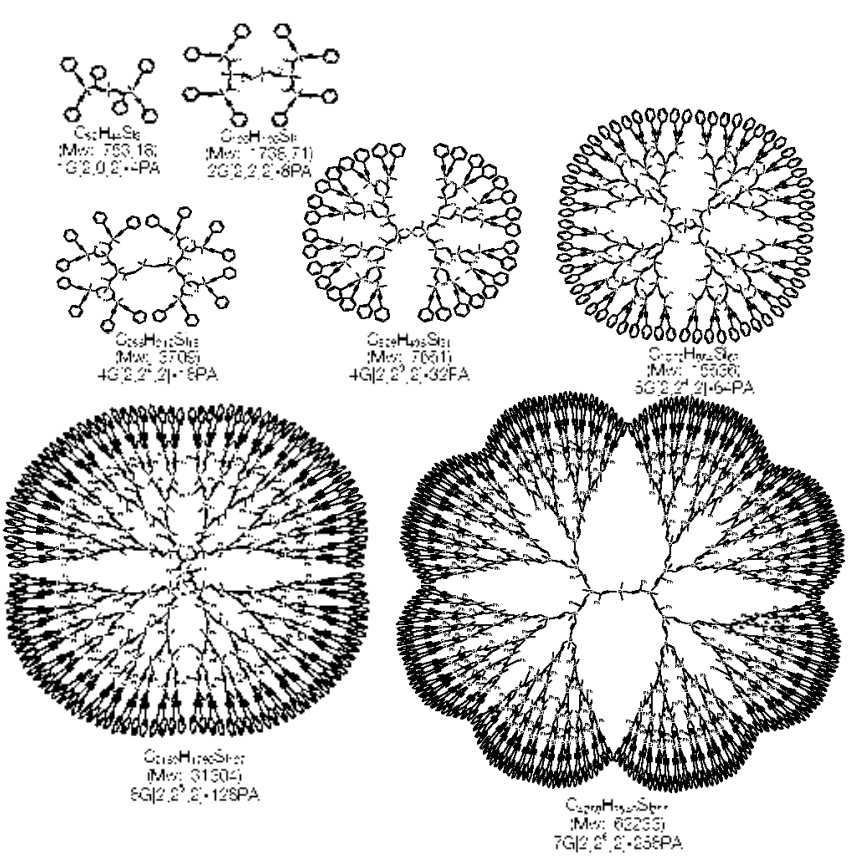

Scheme 2. Schematic structures of bis(phenylethynyl)methylsily] substituted dendrimers $n G\left[2,2^{n-1}, 2\right]^{-} 2^{1 \mathrm{n}-1}$ PA type $\left(\mathrm{n}=1 \sim 7,1^{\mathrm{nt}}\right.$ to $7^{\mathrm{th}}$ generation i in plane. 
syntheses of $7 \mathrm{G}\left[2.2^{6} .2\right]-2^{8} \mathrm{PA}$ having 256 phenylacethylenyl groups is confirmed unequivocally. but no syntheses of $8 \mathrm{G}[2$. $\left.2^{2} \cdot 2\right]-2^{9} \mathrm{PA}$ can be proved by NMR and GPC. It indicates that $7 \mathrm{G}\left[2,2^{6}, 2\right]-2^{8} \mathrm{PA}$ can afford enough space for 256 phenylacethỵlenyl groups. but $8 \mathrm{G}\left[2,2^{2}, 2\right]-2^{9} \mathrm{PA}$ have never formed the unified periphery due to its space not enough for 512 pheny lacethy lenyl groups.

Preparation of anthracene dendrimers (nG $\left[2,2^{n-1}, 2\right]-2^{n+1}$ AN; Type B). ${ }^{{ }^{5 c}}$ The 9-anthracemethoxy groups are introduced into the dendritic periphery by the reaction of Cl-Si bonds of $n G\left[2.2^{n-1} \cdot 2\right]-2^{n+1} \mathrm{Cl}(\mathrm{n}=1 \sim 7)$ with 9-(hydrosymethyl)anthracene in toluene in the presence of TMEDA. After removing TMEDA-HCl salt from the mixture, each dendrimer of $\mathrm{nG}$ $\left[2,2^{1 n-1} \cdot 2\right]-2^{1 n-1}$ AN $(\mathrm{n}=1 \sim 7$ ) having 9-anthracenemethy loxy groups is purified and isolated quantitatively by silica geltoluene/hexane column chromatography. They have been characterized by ${ }^{1} \mathrm{H}$ and ${ }^{12} \mathrm{C}$ NMR. MALDI mass. GPC, and elemental analysis. From MALDI mass spectrum of the respective dendrimer $n G\left[2.2^{n-1} \cdot 2\right]-2^{n-1} A N(n=1$ to 4 ) the molecular ion peak of each dendrimer is observed with its characteristic fragmentation ion peaks. GPC of the respective dendrimer $n G\left[2.2^{1 n \cdot l}, 2\right]-2^{n-l}$ AN $(n=1 \sim 7)$ shows very narrow peak with PDI (polydisperse index) value close to 1.00 at shorter retention time according to increasing its generation (Fig. 2). No synthesis of $8 \mathrm{G}\left[2.2^{2} .2\right]-2^{9} \mathrm{AN}$ is proved by GPC chromatography mainly due to its space not enough for 512 9-antluracenemethosy groups.

Preparation of benzoxazole dendimers ( $\mathrm{nG}\left[2,2^{\mathrm{n}-1}, 1\right]-2^{\mathrm{n}} \mathrm{BO}$; Type C). 2-Benzoxazole-phenyloxy group is bulkier than 9-anthracenemethoxy or pheny lacethylenly group. therefore only one chlorine atom of dichloromethỵlsilỵl group can be substituted by 2-benzoxazoyl-pheny loxy group even at the $1^{\text {st }}$

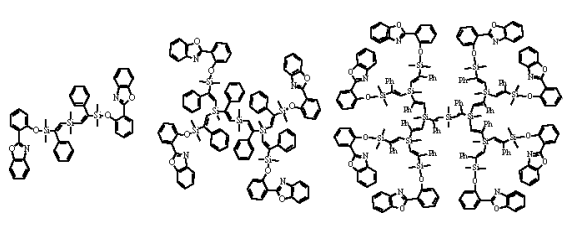

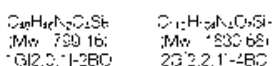

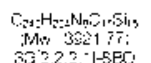

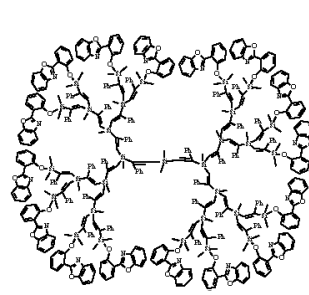

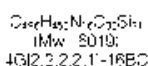
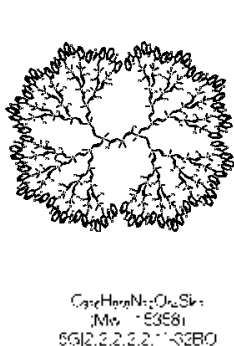
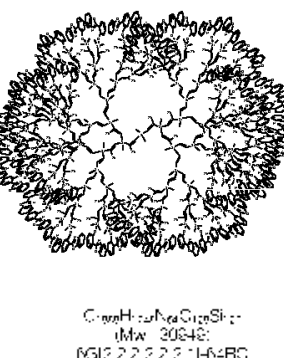

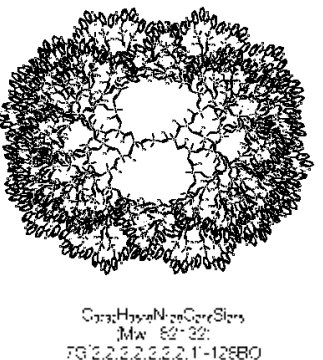

Scheme 3. Schematic structures of (2-benzoxazolyl-2-pheny loxy-dimethylsilyl substituted dendrimers nG $\left[2.2^{\text {itt }] \text { j }}, 1\right]-2^{\text {th }} \mathrm{BO}$ type $\left(\mathrm{n}=1 \sim 7,1^{\text {st }}\right.$ to $7^{\text {th }}$ generation) in plane.

Table 1. Data of GPC and PL for 3 types of dendriners; $n G\left[2,2^{n-1}, 2\right]-2^{n+1} P A, n G\left[2,2^{n-1}, 2\right]-2^{i n+1)} A N$, and $n G\left[2,2^{(n-l)}, 1\right]-2^{n} B O(n=1 \sim 7)$

\begin{tabular}{|c|c|c|c|c|}
\hline $\begin{array}{c}\text { Dendrimers" } \\
\text { (No of Functions) }\end{array}$ & $\begin{array}{l}\text { Fonnula } \\
\left(\mathrm{M}_{w}\right)\end{array}$ & $\begin{array}{c}\mathrm{GPC}^{t^{\prime}} \\
\mathrm{PDI}\left(\mathrm{M}_{1} / \mathrm{M}_{\mathrm{r}}\right) / \mathrm{Rt}(\mathrm{min})\end{array}$ & $\begin{array}{c}\mathrm{PL}^{c} \\
h_{\max }(\mathrm{nm})\end{array}$ & $\mathrm{R}^{d}$ \\
\hline $1 \mathrm{G}[2,2]-4 \mathrm{PA}$ & $\mathrm{C}_{5} \mathrm{H}_{44} \mathrm{Si}_{3}(753.2)$ & $1.01(753.739) / 19.66$ & 418 & \\
\hline $2 \mathrm{G}[2,2,2]-8 \mathrm{PA}$ & $\mathrm{C}_{1: 0} \mathrm{H}_{101 / 1} \mathrm{Si}-(1738)$ & $1.01(1209.1188) / 18.76$ & 418 & \\
\hline $3 \mathrm{G}[2,2,2,2]-16 \mathrm{PA}$ & $\mathrm{C}_{356} \mathrm{H}_{212} \mathrm{Si}_{15}(3709)$ & $1.03(3166.3069) / 17.25$ & 418 & \\
\hline $4 \mathrm{G}[2,2,2,2,2]-32 \mathrm{PA}$ & $\mathrm{C}_{5 \rightarrow 1} \mathrm{H}_{430} \mathrm{Si}_{31}(7651)$ & $1.04(52925075) / 16.35$ & 418 & \\
\hline $5 \mathrm{G}[2,2,2,2,2,2]-64 \mathrm{PA}$ & $\mathrm{C}_{10 \div 3} \mathrm{H}_{884} \mathrm{Si}_{63}(15536)$ & $1.04(11226.10709) / 16.02$ & 418 & \\
\hline $6 \mathrm{G}[2,2,2,2,2,2,2]-128 \mathrm{PA}$ & $\mathrm{C}_{2150} \mathrm{H}_{1: 80} \mathrm{Si}_{12}(31304)$ & $1.07(1+7+7.13774) / 15.54$ & 418 & \\
\hline $7 \mathrm{G}[2,2,2,2,2,2,2,2]-256 \mathrm{PA}$ & $\mathrm{C}_{+288} \mathrm{H}_{3510} \mathrm{Si}_{2 \leq s}(62233)$ & $1.05(17109 \cdot 16165 \mathrm{y} / 15.15$ & 418 & \\
\hline $1 \mathrm{G}[2,2]-4 \mathrm{AN}$ & $\mathrm{C}_{80} \mathrm{H}_{68} \mathrm{O}_{4} \mathrm{Si}_{3}(1177)$ & $1.00(783: 775) / 19.32$ & $349,415,439$ & \\
\hline $2 \mathrm{G}[2,2,2]-8 \mathrm{AN}$ & $\mathrm{C}_{175} \mathrm{H}_{148} \mathrm{O}_{8} \mathrm{Si}-(2587)$ & $1.02(1655 / 1612) / 18.30$ & $349,415,439$ & \\
\hline $3 \mathrm{G}[2,2,2,2]-16 \mathrm{AN}$ & $\mathrm{C}_{258} \mathrm{H}_{308} \mathrm{O}_{15} \mathrm{Si}_{15}(5407)$ & $1.04(5143: 4935 \mathrm{j} / 17.16$ & $349,415,439$ & \\
\hline $4 \mathrm{G}[2,2,2,2,2]-32 \mathrm{AN}$ & $\mathrm{C}_{-3}=\mathrm{H}_{128} \mathrm{O}_{3 \leq} \mathrm{Si}_{31}(11048)$ & $1.01(9843: 9743) / 16.54$ & $349,415,439$ & \\
\hline $5 \mathrm{G}[2,2,2,2,2,2]-64 \mathrm{AN}$ & $\mathrm{C}_{1 \leqslant 00} \mathrm{H}_{12,8} \mathrm{O}_{64} \mathrm{Si} 3(22328)$ & $1.03(12675: 12295) / 15.97$ & $349,415,439$ & \\
\hline $6 \mathrm{G}[2,2,2,2,2,2,2]-128 \mathrm{AN}$ & $\mathrm{C}_{30 \leqslant 6} \mathrm{H}_{2 \leqslant 48} \mathrm{O}_{1 \leqslant 8} \mathrm{Si}_{12}(44888)$ & $1.05(1531514474) / 15.45$ & $349,415,439$ & \\
\hline $7 \mathrm{G}[2,2,2,2,2,2,2,2]-256 \mathrm{AN}$ & $\mathrm{C}_{61 \leqslant 3} \mathrm{H}_{\leqslant 1188} \mathrm{O}_{2 \leqslant 6} \mathrm{Si}_{2 \leq s}(90009)$ & $1.06(17008.16009) / 15.30$ & $349,415,439$ & \\
\hline $1 \mathrm{G}[2,1]-2 \mathrm{BO}$ & $\mathrm{C}_{4 \$ 8} \mathrm{H}_{8 \mathrm{r}} \mathrm{N}_{2} \mathrm{O}_{4} \mathrm{Si}:(799.2)$ & $1.01(682.674) / 19.93$ & 475 & \\
\hline $2 \mathrm{G}[2,2,1]-4 \mathrm{BO}$ & $\mathrm{C}_{112} \mathrm{H}_{10+} \mathrm{N}_{4} \mathrm{O}_{8} \mathrm{Si}=(1830)$ & $1.02(11101082) / 18.92$ & 475 & \\
\hline $3 \mathrm{G}[2,2,2,1]-8 \mathrm{BO}$ & $\mathrm{C}_{2+2} \mathrm{H}_{224} \mathrm{~N}_{8} \mathrm{O}_{16} \mathrm{Sil}_{5}(3921)$ & $1.02(3+723389 \mathrm{j} / 17.72$ & 475 & \\
\hline $4 \mathrm{G}[2,2,2,2,1]-16 \mathrm{BO}$ & $\mathrm{C}_{406} \mathrm{H}_{45_{2}} \mathrm{~N}_{15} \mathrm{O}_{32} \mathrm{Si}_{31}(8019)$ & $1.00(55885562 \mathrm{~g} / 16.84$ & $357 / 475$ & $\mathrm{~T}$ \\
\hline $5 \mathrm{G}[2,2,2,2,2,1]-324 \mathrm{BO}$ & $\mathrm{C}_{036} \mathrm{H}_{868} \mathrm{~N}_{32} \mathrm{O}_{64} \mathrm{Si}_{62}(15358)$ & $1.04(1130270803) / 15.95$ & 357 & \\
\hline $6 \mathrm{G}[2,2,2,2,2,2,1]-64 \mathrm{BO}$ & 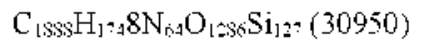 & $1.06(12538: 11775 \mathrm{y} / 15.53$ & 357 & \\
\hline $7 \mathrm{G}[2,2,2,2,2,2,2,1]-128 \mathrm{BO}$ & $\mathrm{C}_{3-4)} \mathrm{H}_{3 \leq 1) 8} \mathrm{~N}_{138} \mathrm{O}_{2 \leq 5} \mathrm{Si}_{-34}(62132)$ & $1.01(1+898 \cdot 1+623 y / 15.3$ & 357 & \\
\hline
\end{tabular}

${ }^{a} \mathrm{PA}$ : Phenylethynylsilane terminated dendrimer. AN: Anthracene terninated dendrimer. BO; Benzoxazole terminated dendrimer, "GPC: Gel Permeation Chromatography, "PL: Photoluminescence, dendrimers with anthracene (AN) have three masimal peaks. 'T: Dendrimers show blue shift in PL data. 
generation of $1 \mathrm{G}|2.0 .1|-2 \mathrm{Cl}$. Therefore another dendrimer of $\mathrm{nG}\left|2 \cdot 2^{\mathrm{n}-1} \cdot 1\right|-2^{\mathrm{n}} \mathrm{Cl}(\mathrm{n}=\mathrm{I} \sim 7)($ ty pe $\mathrm{C})$ is designed and prepared by the hydrosilation of pheny lacety lenyl group with dimethylchlorosilane instead of dichloromethy Isilane. One 2-bentoxazole-pheny loxy group is substituted on cach silicon atom of the dendrimer $\mathrm{nG}\left|2.2^{\mathrm{n}]} \cdot 1\right|-2^{\mathrm{n}} \mathrm{Cl}(\mathrm{n}=1 \sim 7)$ by the reaction of Cl-Si bond with 2-(2-hydroxy phenyl)benzoxazole in the presence of TMEDA. From the reacion nixture dendrimer of $\mathrm{nG}\left|2.2^{\mathrm{n}-1} \cdot 1\right|-2^{\mathrm{n}} \mathrm{BO}(\mathrm{n}=1 \sim 7)$ is purified and isolated quantitatively by silica gel-toluene chromatography, Each dendrimer is characterized by ' $\mathrm{H}$ and ${ }^{1.3} \mathrm{C}$ NMR. MALDI-MS. and elemcntal analy sis. All dendrimers of $\left.n G \mid 2.2^{n-1} \cdot 1\right]-2^{n} \mathrm{BO}$ (Scheme 3) are soluble in organic solvent such as toluene. diethyl ether. and chlorolorm. The moleculelarity of the respective dendrimer $\mathrm{nG}\left|2.2^{n} .1\right|-2^{n} \mathrm{BO}(\mathrm{n}=1 \sim 7)$ is determined by GPC. in which very narrow peak with PDI value close to 1.00 at shorter retention time according to increasing its generation (Fig. 2).

Measurements of the unified properties for cartosilane dendrimers: For the preparation and identification of higher generation dendrimer it has been very critical and controversial to measure its unimolecularity or purity. The MALDI mass spectroscopy provides valuable information for the unimolecularity of lower generation dendrimer; nonetheless generally it gives no conclusive information for its purity. Therefore in this work GPC has been chosen to study the moleculclarity of the dendrimer of $n G\left[2 \cdot 2^{n-1} \cdot 2\right]-2^{n-1} P A(n=1 ~ \sim$ 7) (Fig. 1 and Table 1).

The GPC chromatogram in Fig. I shows very narrow peaks corresponding to the respective generation of the dendrimers. As same as linear polymers. the peak of heavier dendrimer comes first in GPC. The first peak from left is that of the highest generation dendrimer. $7 \mathrm{G}\left|2,2^{6}, 2\right|-2^{k} \mathrm{PA}$, and the last peak from lefi is that of the lowest generation dendrimer. $I G \mid 2,0.21-2^{2} \mathrm{PA}$. Most significant feature of the chromatogram is that cach peak has no shoulder in the left and the right side of the peak for the $1^{\text {st }}$ to the $5^{\text {th }}$ generation dendrimers in Figure 1 and 2. On the other hand for the $6^{\text {th }}$ and/or $7^{\text {th }}$ generation of them a litile shoulder can be recognized in the only left side of each peak. The shoulder in the left side of the peak must be conte from the corresponding dendrimer having impurities. which are stuck between its branches. 1 is plausible that higher generation dendrimer has more space to capture any impurity between its branches. Nevertheless no shoulder in the right side of cach peak is obsered. it indicates that the purified each dendrimer has no structural defect on its branches or periphery:

In addition the PDI values of the peaks are very close to 1.00 in the range from 1.00 to 1.07 . It indicates that the calculated molecular weight from GPC is very little greater than that of the dendrimer with no defect $\left(M_{n} \geq M_{n}\right)$. From these results it seems to be reasonable that the closer to 1.00 the PDI value of dendrimer is observed. the purer dendrimer is oblained. However. in the preparation of the $8^{\text {th. }}$ generation $\left.8 \mathrm{G} \mid 2.2^{7} .2\right]-2^{\circ} \mathrm{PA}$ no narron peak in GPC cliromatogram is observed.

In conclusion. three types of dendrimer, $n G\left|2.2^{n-1} \cdot 2\right|-2^{n \cdot 1}$ PA for phenvilethỵyl group substituted dendrimer. $\mathrm{nG} \mid 2.2^{n-1}$.

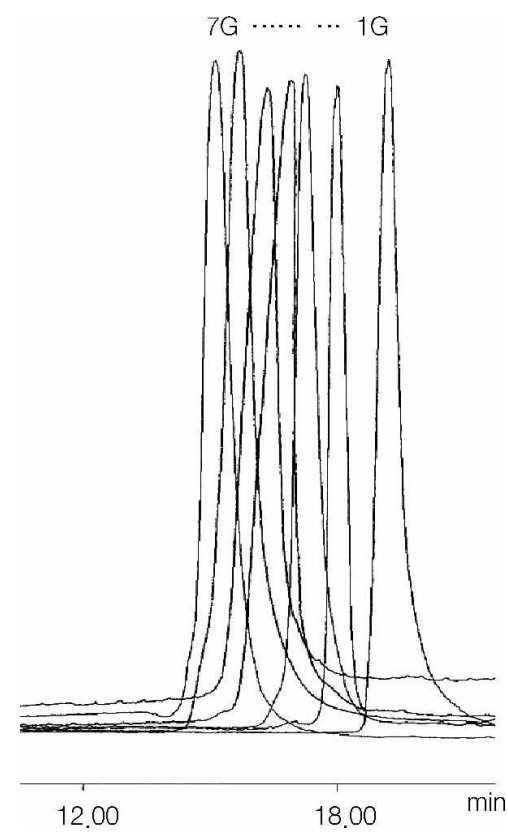

Figure 1. GPC diagram for phens let hy nu lisiane (eminaled dendrimers $n G \mid 2,2^{11} \cdot 21-2^{11 \cdot 1} \mathrm{P} \wedge(\mathrm{n}=1-7)$
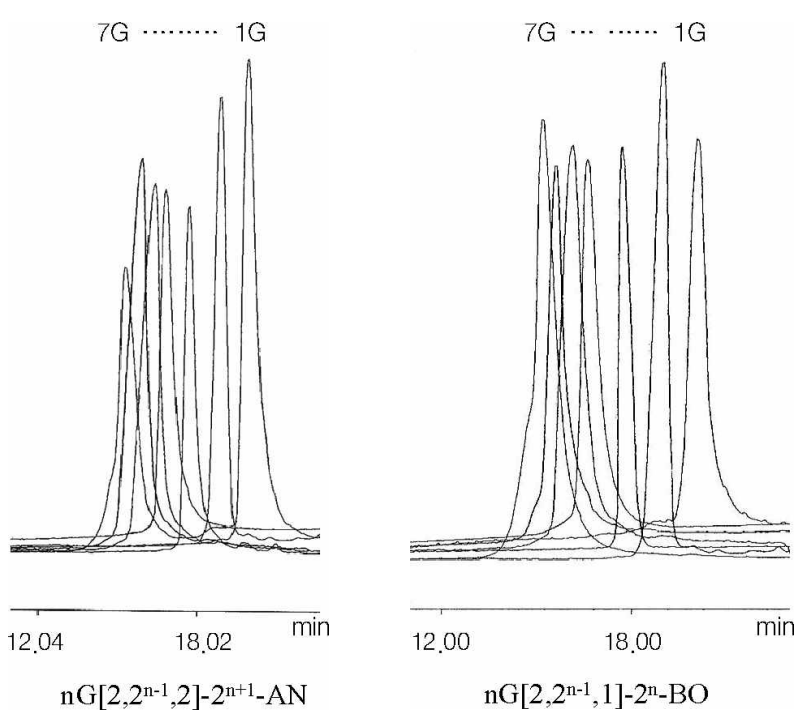

Figure 2. GPC diagram for anthraene teminated dendrimess nG

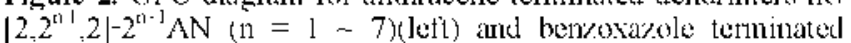

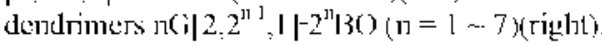

$2 \mid-2^{\mathrm{n} \cdot \mathrm{l}}$ AN for 9-anthracenemethoxy group substituted dendrimer and $n \mathrm{G}\left|2.2^{n-1} .1\right|-2^{\prime \prime} \mathrm{BO}$ for 2-ben\%oxa\%olc-phenoxy group substituted dendrimer. have synthesized up to the $7^{\text {ih }}$ generation. after which no cnough space secms to be available lor the incoming 2-ben/oxazole-phenoxy group on the periphery:

Measurements of photoluminescent projerties of the dendimes: The PL spectra of $n G\left|2.2^{n-1} \cdot 2\right|-2^{n \cdot l} P A(n=1 \sim 7)$ show one $\lambda_{\max }$ at the wavelength of 418 nm (Figure 3 ). The PL

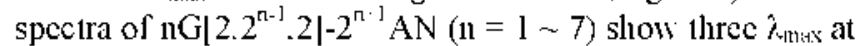
the wavelength of 349 . 415 and $439 \mathrm{~nm}$. those are charac- 

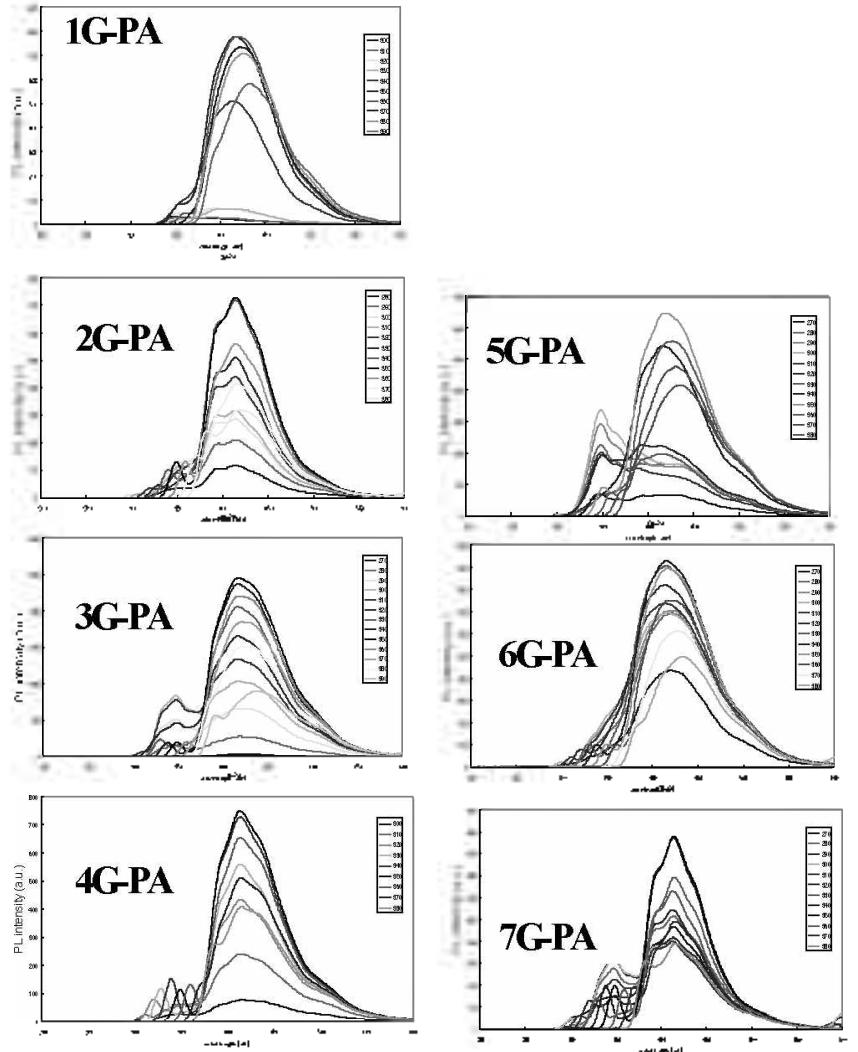

Figure 3. Photoluminescent spectra of the dendrimers substituted by phenylethynyl group $\mathrm{nG}\left[2,2^{\mathrm{n}-1}, 2\right]-2^{\mathrm{n}^{+1}} \mathrm{PA}(\mathrm{n}=1 \sim 7)$.
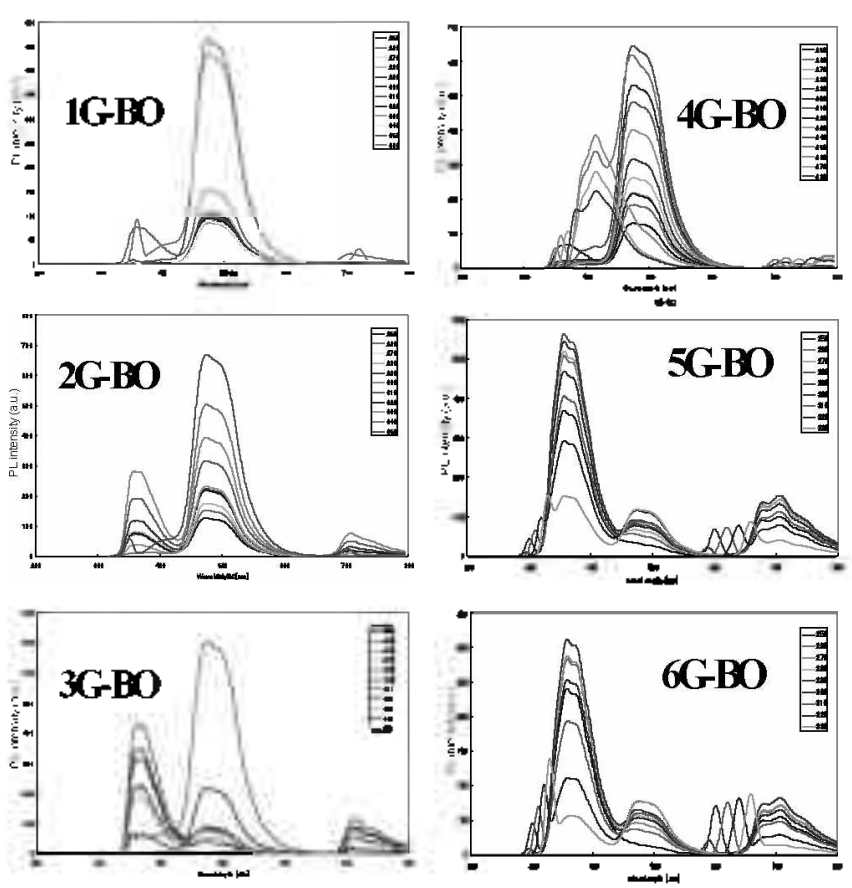

Figure 5. Photoluminescent spectra of dendrimers substituted by 2bezoxazole-phenoxy group $\mathrm{nO}\left[2,2^{\mathrm{n}-1}, 1\right]-2^{\mathrm{n}} \mathrm{BO}(\mathrm{n}=1 \sim 6)$.

teristic peaks of anthracene (Figure 4). With increasing generation of the dendrimer the absorption of $\lambda_{\text {max }}$ shows no significant change the intensity of $\hat{z}_{\text {max }}$ simply depends on the

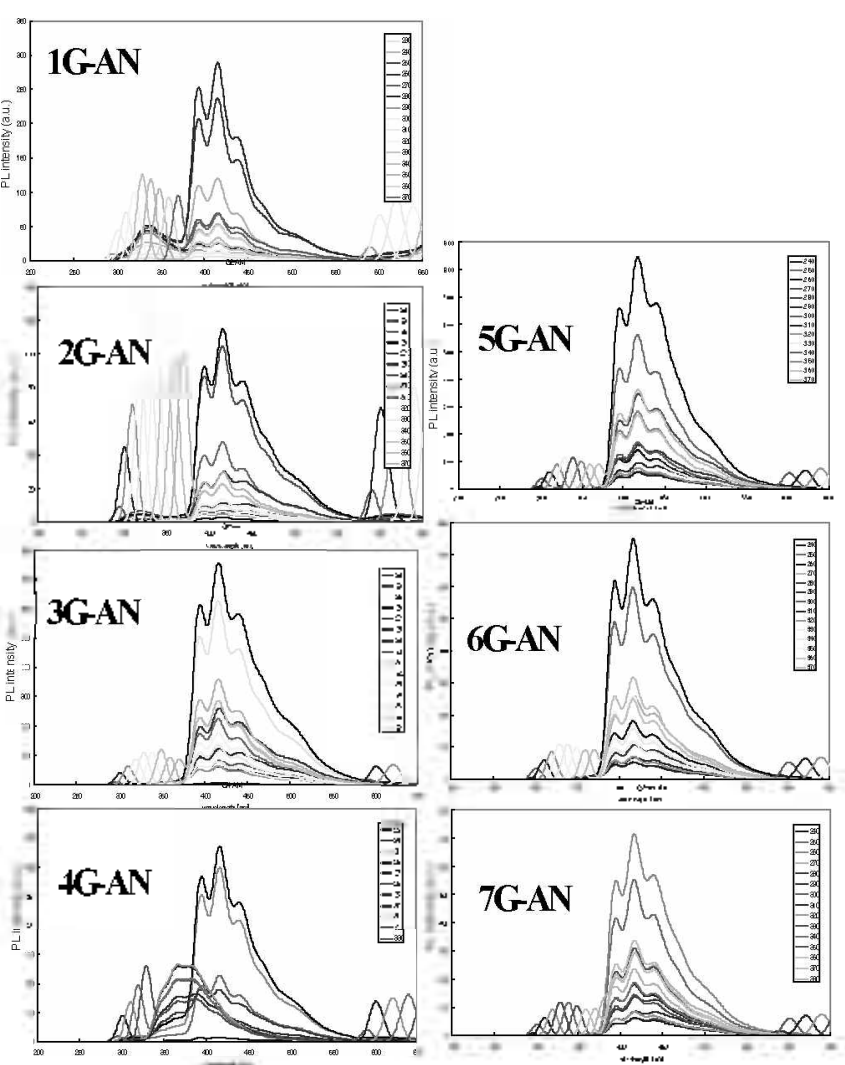

Figure 4. Photoluminescent spectra of dendrimers substituted by anthracenemethosy group $n G\left[2,2^{n-1}, 2\right]-2^{n^{-1}} \mathrm{AN}(\mathrm{n}=1 \sim 7)$.

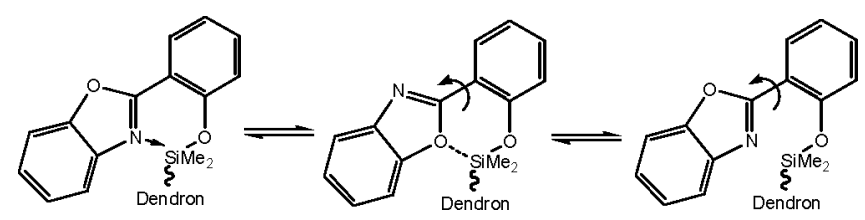

Orientation ir lower generation

Orientaior in higher generation

Scheme 4. Suggested mechanism for blue-shift of $n \mathrm{G}\left[2,2^{\mathrm{n}-1}, 1\right]-2^{\mathrm{n}} \mathrm{BO}$ dendrimers.

numbers of chromophores attached to the dendrimer. It indicates that the number of peripheral triple and double bonds inside dendrimer has no influence on PL spectrum of the dendrimer.

The PL spectra of $\mathrm{nG}\left[2,2^{17-1} \cdot 1\right]-2^{\mathrm{n}} \mathrm{BO}(\mathrm{n}=1 \sim 6)$ show one smaller $\lambda_{\text {max }}$ at the wavelength of 347 and the other bigger $\lambda_{\text {max }}$ at the wavelength of $475 \mathrm{~nm}$. Unexpectedly the intensity of the smaller $\lambda_{\text {max }}$ at the wavelength of $347 \mathrm{~nm}$ is increased and the intensity of the bigger $\lambda_{\max }$ at the wavelength of $475 \mathrm{~nm}$ is decreased with increasing the generation of $\mathrm{nG}\left[2.2^{13-1} \cdot 1\right]$ $2^{\mathrm{th}} \mathrm{BO}$, from the $1^{\mathrm{st}}$ to the $6^{\mathrm{th}}$ generation. In the end the intensity of $\lambda_{\max }$ at the wavelength of $347 \mathrm{~cm}$ is bigger than that of $\lambda_{\max }$ at the wavelength of $475 \mathrm{~nm}$ for the $5^{\mathrm{dh}}$ and the $6^{\mathrm{dh}}$ generation of $\mathrm{nG}\left[2.2^{1 \mathrm{n}-1} \cdot 1\right]-2^{\mathrm{n}} \mathrm{BO}(\mathrm{n}=5,6)$. It is plausible that the space around $\mathrm{Si}$ atom becomes smaller with increasing the generation. For the lower generation dendrimer there is enough space around $\mathrm{Si}$ atom for the bulky 2-bezoxazolephenoxy group to be planar geometry. in which nitrogen as well as oxygen atonts of the 2-bezoxazole-phenoxy group can coordinate 
equatorially to Si atom. However for the higher generation dendrimer 2-bezoxazole-phenoxy group is too bulky to move around $\mathrm{Si}$ atom. therefore the benzoxazole and phenyl groups rotate to minimize their bulkiness (Scheme 4 ).

Acknowledgments. This work was supported by grant No (R01-2006-000-11120-0) from the Basic Research Program Korea Science and Engineering Foundation of Ministry of Science \& Technology and personally supported by Korean Research Foundation (KRF-2006-351-C00015).

\section{References}

1. Buhleier, E.: Wener, W: Vögtle, F. Smthesis 1978, 155.

2. Issbent, J:- Moore, R.; Vögtle, F. Aliegew. Chem. Iht Ed Engl. $1994,33,2413$.

3. DeMattei, C. R.; Huang, B.; Tomalia, D. A. Nano Lett. 2004, 4, 771.

4. Zhao, M.: Helms, B.: Slonkina, E.: Friedle, S: Lee, D.: DuBios, . T: Hednan, B.; Hodgson, K. O.: Fréchet, J. M.; Lippard, S. I. J. Ant. Chent. Soc $\mathbf{2 0 0 8}, 130,4352$

5. (a) Kim, C: Park, J. J. Orgunomet. Chem. 2001, 269, 194. (b) Kinn, C. Jeong, K. Tung, I. J. Polm. Sci.: Part t: Polm. Chem. 2000, 38, 2749. (c) Kim, C.; Kim, H.; Park, K. J. Organomet. Chen. 2003, 667, 96 .

6. Schalley, C. A.; Vogtle, F. Dendrimer I: Functional and Hyperbranched Building Block. Photophysical Properties. Applications in Materials and Life Science: Sprunger Verlag: Berlin, 2003
7. Lee, W. I.: Bae, Y.: Bard, A. J. J. Am. Chem. Soc. 2004, 126. 8358.

8. Tang, S.; Martinez, L. T.; Sharma, A.; Chai, M. Org. Lett. 2006, 8. 4421

9. Wilson, O. M: Scott, R. W. T.; Garcia-Martinez, J. C.; Crooks, R. M. Chem Mater 2004, 16, 4202 .

10. Nijuis, C. A.; Huskens, T; Reinhoudt, D. N. J. Am. Chem. Soc. 2004, 126, 12266 .

11. Kim, K. D.: Kim, D. S.: Kim, C.: Song, C. K. Jap. J .Appl Phy $2008,+76,496$.

12. Yoon, H. C.; Shin, H. K.; Kim, C.; Kwon, Y. S. Synt. Het. 2003 , 137,1427

13. Cho, B.-K; Jain, A.; Mahajan, S.; Ow; H.; Gruner, S. M: Wiesner, U. J.Am. Chem. Soc. 2004, 126,4070.

14. Kim, C.; Kim, H. Symthesis 2005, 381.

15. Kim, C; Lim, K.-I; Song, C. K. J. Orgonontet. Chem. 2005, 690,3278

$16 \mathrm{Kim}, \mathrm{C} . ; \mathrm{Kim}, \mathrm{H} ;$ Park, K. J. Organomet Chem. 2005, 690, 4794.

17. Hirayama, Y: Nakamura, T; Uehara, S.; Sakamoto, $Y$; Yamaguchi, K.; Sei, Y.; Iwamura, M. Org. Lett. 2005, 7, 525.

18. Müller, R.: Laschober, C.: Szymanski, W. W.: Allmaier, G. Hacrontolecules $\mathbf{2 0 0 7}, 40,5599$.

19. Krupková, A: Cenmak, J.: Walterová, Z: Horský, J. Amal. Chent. 2007, 79, 1639

20. Bu, L.; Nonidez, W. K.; Mays, T. W. Mfacromolecules 2000, 33 , 4445.

21. Kim, C; Seo, W; Oh, M.-T. Bull. Korean Chem, Soc. 2007, 28, 1963. 